\title{
Mitochondrion-Directed Nanoparticles Loaded with a Natural Compound and a microRNA for Promoting Cancer Cell Death via the Modulation of Tumor Metabolism and Mitochondrial Dynamics
}

\author{
Yu-Li Lo ${ }^{1,2, * \mathbb{B}}$, Chen-Shen Wang ${ }^{1}$, Yen-Chun Chen ${ }^{1}$, Tse-Yuan Wang ${ }^{1}$, Yih-Hsin Chang ${ }^{3}$, \\ Chun-Jung Chen ${ }^{4}$ and Ching-Ping Yang ${ }^{4}($ ) \\ 1 Institute of Pharmacology, National Yang-Ming University, Taipei 112, Taiwan; \\ peach50505@gmail.com (C.-S.W.); mikechen023@gmail.com (Y.-C.C.); alanwang.twn@gmail.com (T.-Y.W.) \\ 2 Center for Advanced Pharmaceutics and Drug Delivery Research, National Yang-Ming University, \\ Taipei 112, Taiwan \\ 3 Department of Biotechnology and Laboratory Science in Medicine, National Yang-Ming University, \\ Taipei 112, Taiwan; cyh@ym.edu.tw \\ 4 Department of Medical Research, Taichung Veterans General Hospital, Taichung City 407, Taiwan; \\ cjchen@vghtc.gov.tw (C.-J.C.); milkygp@gmail.com (C.-P.Y.) \\ * Correspondence: yulilo@ym.edu.tw; Tel.: +88-62-2826-7000 (ext. 7095)
}

Received: 28 July 2020; Accepted: 6 August 2020; Published: 11 August 2020

\begin{abstract}
Mitochondrial dysfunction may cause cancer and metabolic syndrome. Ellagic acid (abbreviated as E), a phytochemical, possesses anticancer activity. MicroRNA 125 (miR-125) may regulate metabolism. However, E has low aqueous solubility, and miR-125 is unstable in a biological fluid. Hence, this study aimed to develop nanoparticle formulations for the co-treatment of miR-125 and E. These nanoparticles were modified with one mitochondrion-directed peptide and a tumor-targeted ligand, and their modulating effects on mitochondrial dysfunction, antitumor efficacy, and safety in head and neck cancer (HNC) were evaluated. Results revealed that miR-125- and E-loaded nanoparticles effectively targeted cancer cells and intracellular mitochondria. The co-treatment significantly altered cellular bioenergetics, lipid, and glucose metabolism in human tongue squamous carcinoma SAS cells. This combination therapy also regulated protein expression associated with bioenergenesis and mitochondrial dynamics. These formulations also modulated multiple pathways of tumor metabolism, apoptosis, resistance, and metastasis in SAS cells. In vivo mouse experiments showed that the combined treatment of miR-125 and E nanoparticles exhibited significant hypoglycemic and hypolipidemic effects. The combinatorial therapy of $\mathrm{E}$ and miR- 125 nanoparticles effectively reduced SAS tumor growth. To our best knowledge, this prospective study provided a basis for combining miRNA with a natural compound in nanoformulations to regulate mitochondrial dysfunction and energy metabolism associated with cancer.
\end{abstract}

Keywords: EGFR targeting; mitochondrion-directed nanoparticles; natural compound; microRNA; mitochondrial dynamics; cancer

\section{Introduction}

Epithelial-mesenchymal transition (EMT) and chemoresistance are two major challenges of the successful treatment of various tumors, such as oral squamous cell carcinoma (OSCC), a type of head and neck cancer (HNC) [1]. Alterations in mitochondrial dynamics and mitophagy are associated with the chemoresistance and EMT of tumor cells [2]. Mitochondrial dynamics is misbalanced when the 
mitochondria change from a highly organized and elongated network (mitochondrial fusion) into a fragmented and punctate form (fission) [3]. Furthermore, the suppression of mitochondrial fission is involved in cellular survival, neoplastic progression, and drug resistance [4].

Mitophagy may act as a pro-survival method of cells under inflammation by maintaining the mitochondrial dynamic balance. Mitophagy has become a potential anticancer target by triggering mitochondrial dysfunction in cancer cells because of the imperative role of mitophagy on mitochondrial homeostasis and cell survival. Remarkably, phosphatase and tensin homolog (PTEN)-induced putative kinase 1 (PINK1)/Parkin-related mitophagy is usually activated by mitochondrial damage [5]. Dysfunctional mitochondria with reduced mitochondrial membrane potential (MMP) may activate PINK1 and then trigger Parkin to be overexpressed on the surfaces of the mitochondria, thereby inducing mitophagy [6]. PINK1/Parkin- dependent mitophagy plays an important role in mitochondrial homeostasis [7], and the modulation of PINK1/Parkin-mediated mitophagy alters the sensitivity of multidrug-resistant cancer cells to anticancer agents [8]. Accumulating evidence has indicated that the inhibition of Parkin-related mitophagy increases cancer cell death [9]. Recently, tanshinone IIA, a potential antitumor agent, has been found to suppress the adenosine monophosphate-activated protein kinase (AMPK) pathway and inactivate Parkin, thus decreasing mitophagy activity and enhancing colorectal cancer (CRC) cell apoptosis [10]. A similar series of events occurs in various tumor types [6,11]. Nevertheless, contradictory findings have suggested that the activation of mitophagy may improve the cytotoxicity of drugs against various cancers [12]. For example, zinc oxide nanoparticles increase the intracellular levels of reactive oxygen species (ROS) and reduce the MMP, but these nanoparticles stimulate the PINK1/Parkin-mediated mitophagy to induce the anticancer activity in tongue cancer CAL 27 cells [13]. Thus, the role of mitophagy in cancer progression or inhibition should be clarified.

Ellagic acid (abbreviated as E), a natural polyphenolic phytochemical, can be isolated from edible plants, algae, and mushroom [14]. Recent studies have shown that E possesses anticancer, anti-inflammatory, and antioxidative activities [15]. Consistently, E may reduce metabolic syndromes caused by high-fat diets (HFDs) in rats [16]. Furthermore, a natural product extract containing E increases the sensitivity of CRC cells to 5 -fluorouracil (5-FU) therapy by triggering a ROS-mediated and mitochondrion-associated apoptotic pathway via the induction of mitochondrial dysfunction and autophagy [17].

MicroRNA 125 (miR-125), a miRNA of 22 nucleotides located at chromosome 19q13, is found to have commonly lower expression in several tumor types, including CRC, HNC, ovarian cancer, lung cancer, and medulloblastoma [18-20]. miR-125 suppresses CRC cell growth by targeting and decreasing the expression of vascular endothelial growth factor, triggering CRC cell apoptosis [19]. Interestingly, oncogenic long noncoding RNA may regulate glycolysis by binding to miR-125 and affect the progression of esophageal squamous cell carcinoma cells [20]. miR-125 modulates the gene expression levels in lipid and glucose utilization [21]. Additionally, miR-125 may affect oxygen consumption and mitochondrial DNA content, modulating mitochondrial bioenergenesis and respiration [21]. These studies have suggested the critical role of miR-125 in regulating lipid and glucose metabolism, mitochondriogenesis, and cancer cell apoptosis.

However, miRNA is unstable in biological systems because of its rapid degradation by endonucleases and exonucleases and fast elimination from the body. Furthermore, it experiences difficulty in penetrating the cell membrane because of its negative charge [22]. Hence, we proposed cationic low-toxicity solid lipid nanoparticles (SLN) to prevent miR from degradation and clearance and reach intracellular target sites. SLN, consisting of a cationic lipid and a surfactant, may form a stable mixture with miR-125 via charge attraction. SLN also possesses the characteristics of physical stability and protective property of miRNA [22]. Therefore, we prepared miR-125 in SLN to function as a combination therapeutic agent to enhance the effect of $E$ as an effective antineoplastic agent by regulating mitochondrial dynamics in poorly differentiated human squamous SAS cell carcinoma of the tongue (one OSCC cell line). 
Furthermore, E has poor aqueous solubility, rapid body clearance, and low bioavailability. In addition, its clinical application is limited by its fast metabolism and enzymatic degradation [23]. Thus, we designed lipid-polymer nanoparticles (LPNs) to improve the aforementioned problems of E. LPNs are lipid-based delivery systems of drugs or natural compounds. LPNs are usually composed of a surface phospholipid shell and a biocompatible polymer core, such as poly(lactic-co-glycolic acid; PLGA), an FDA-approved biodegradable polymer [24]. This structure combines the advantages of a lipophilic compound/PLGA nanocore and a lipid shell [25].

Thus, the design of LPN and SLN in the present study may prevent the metabolism and degradation of $E$ and miR-125 and increase their systemic stability to improve the cellular uptake of the encapsulated cargos. Further coupling of LPN and SLN with peptides/ligands of different functions can provide a tumor-specific targeting delivery [26]. Hence, we designed nanoparticles by modifying their surfaces with two functional peptides that targeted cancer cells to improve cellular penetration and subcellular mitochondrial specificity. The targeting of LPN and SLN to specific receptors overexpressed in tumor cells may reduce the toxicity to normal cells [24]. Additionally, the mitochondrion-directed nanoparticles may display the potential to modulate mitochondria-mediated cancer cell death [27].

L peptide is screened from the phage display peptide library with good epidermal growth factor receptor (EGFR) binding and tumor targeting characteristics [28]. This peptide also possesses a prototypic CendR motif responsible for triggering extravasation and tumor penetration [28]. K peptide is screened with remarkable mitochondrion and tumor-focused characteristics. Previous studies have demonstrated that $\mathrm{K}$ peptide has a mitochondrial membrane disrupting function and can enhance the intracellular accumulation of nanoparticles via the electrostatic interaction between a lysine group and an anionic tumor cell surface $[29,30]$. The cationic domain of this peptide disrupts the negatively charged mitochondrial membranes, which have a high transmembrane potential [31]. In our study, $K$ peptide was verified in terms of its mitochondrial targeting property and penetrating effect on cancer cells. Hence, the lipid shell of SLN and LPN was modified with L peptide to target EGFR-overexpressing SAS tumors and with M peptide to direct the mitochondria and enhance cancer penetration for SLN-KL and LPN-KL formulations. Furthermore, miR-125 and E were incorporated into SLN-KL and LPN-KL to prepare miR-125/SLN-KL and E/LPN-KL, respectively. Thus, due to the potential roles of miR-125 and $\mathrm{E}$ in regulating mitochondrial dynamics, apoptosis, and migration of cancer cells, we aimed to explore the effects and mechanisms of the combinatorial therapy of miR-125/SLN-KL and E/LPN-KL in suppressing HNC via multiple signaling modulations of tumor energy metabolism, metastasis, and resistance in vitro and in vivo.

\section{Materials and Methods}

\subsection{Materials}

E, lecithin, distearoylphosphatidylethanolamine (DSPE)-polyethyleneglycol molecular weight 2000 (PEG2000), and PLGA were purchased from Sigma-Aldrich (St. Louis, MO, USA). miR-125 and FAM-miR125 were purchased from GenePharma (Shanghai, China). Peptide K and L were bought from Kelowna Biotech (Taipei, Taiwan) at $>95 \%$ purity. Monoclonal antibodies (Mab) of B-cell lymphoma 2 (Bcl-2), Bcl-2 associated X-protein (Bax), $\beta$-catenin, caspase 9, caspase 3, poly (ADP-ribose) polymerase (PARP), receptor-interacting protein (RIP)1, RIP3, Slug, E-cadherin, etc. were bought from Cell Signaling Technology (Beverly, MA, USA), GeneTex (Irvine, CA, USA), Jackson (West Grove, PA, USA), or Abcam (Cambridge, UK), as demonstrated in Supplementary Table S1. All cell culture medium and reagents were obtained from Promega (Madison, WI, USA), Invitrogen (Carlsbad, CA, USA), Gibco BRL (Grand Island, NY, USA), or Hyclone (Logan, UT, USA). All other chemical reagents were purchased from either Merck (Darmstadt, Germany) or Sigma-Aldrich. 


\subsection{Synthesis and Preparation of E/LPN-KL and miR125/SLN-KL}

DSPE-PEG was mixed with peptide $\mathrm{K}$ or $\mathrm{L}$ at a molar ratio 1:1 for $24 \mathrm{~h}$ at room temperature to synthesize K or L-linked lipids. The products were put in a dialysis bag against water. The purified K or L-bound lipids were freeze-dried and detected by matrix-assisted laser desorption ionization time-of-flight mass spectrometry (MALDI-TOF MS; Bruker, Bremen, Germany).

E/LPN-KL was prepared by an o/w emulsion method by dispersing $3 \mathrm{mg} / \mathrm{mL}$ of $\mathrm{mPEG}-\mathrm{DSPE}$ or peptide-conjugated lipid and lecithin in ethanol/water (1:1) solution. E in PLGA (50:50 LA:GA) was prepared at a concentration of $2 \mathrm{mg} / \mathrm{mL}$ and added to the above dispersion dropwise. The resulting dispersion was mixed by sonication for $10 \mathrm{~min}$. The resulting E-loaded nanoparticles were stirred using a centrifugal evaporator to allow the solvent to vaporize completely and then stored at $4{ }^{\circ} \mathrm{C}$.

SLN was prepared by mixing lecithin, 1, 2-dioleoyl-3-trimethylammonium-propane (DOTAP), DSPE-PEG-peptide at a molar ratio of 1:0.1:0.1 in methanol/dichloromethane (2:1). The dispersion was added into Tween 80 solution drop by drop. A miR-125 solution of $100 \mathrm{nM}$ was incorporated into SLN-KL and incubated at room temperature for $30 \mathrm{~min}$ to form a final dispersion of miR125/SLN-KL.

\subsection{Size Distribution and Zeta Potential of $L P N$ and SLN}

The size distribution and zeta potential of LPN and SLN were detected using a Zetasizer nano ZS analyzer (Malvern Instruments Ltd., Malvern, Worcestershire, UK). Data were computed by a Malvern software to acquire the values of the polydispersity index.

\subsection{The Shape and Particle Morphology by Transmission Electron Microscopy (TEM)}

The surface morphology of nanoparticles was observed using a transmission electron microscopy (TEM) system (JEM-2000EXII, Tokyo, Japan). One drop of the sample solution was mounted onto a carbon-coated copper grid and then visualized under TEM.

\subsection{Encapsulation Efficiency (EE\%)}

Unbound $\mathrm{E}$ or miR was separated from the loaded LPN or SLN by centrifugation. E or miR in the filtrate was analyzed by HPLC or NanoDrop (Thermo Fisher, MA, USA). The HPLC system composed of a pump (L7100; Hitachi, Tokyo, Japan), accompanied with an automated injector (L2200), a $5 \mu \mathrm{m}$ LiChrospher column (25 cm long; Merck, Darmstadt, Germany), and an ultraviolet detector (L2400; Hitachi, Tokyo, Japan). The mobile phase included $60 \%$ methanol and water (gradient solvent from 50:50 to 70:30, $v / v$ ) plus o-phosphoric acid and ran at a flow rate of $1 \mathrm{~mL} / \mathrm{min}$. The solvent/water was pre-degassed using a sonicator. The detection wavelength was set at $259 \mathrm{~nm}$.

$\mathrm{EE} \%$ was calculated as the percentage of the amount of $\mathrm{E}$ or miR in LPN or SLN divided by the total amount of added E or miR. EE\% and DL\% were calculated by Equation (1), as shown below.

$$
\mathrm{EE} \%=\left[\left(\mathrm{W}_{\mathrm{e}}-\mathrm{W}_{\mathrm{f}}\right) / \mathrm{W}_{\mathrm{e}}\right] \times 100 \%
$$

where $\mathrm{W}_{\mathrm{e}}$ is the weight of added $\mathrm{E}$ or $\mathrm{miR}$, and $\mathrm{W}_{\mathrm{f}}$ is the weight of $\mathrm{E}$ or miR in the filtrate.

\subsection{Cell Lines}

SAS and normal oral keratinocyte (NOK) cells, obtained from the lab of Professor Muh-Hwa Yang (National Yang-Ming University), were incubated in DMEM containing 10\% FBS and 1\% penicillin/streptomycin at $37{ }^{\circ} \mathrm{C}$ in an incubator of $5 \% \mathrm{CO}_{2}$ and $90 \%$ relative humidity. All the cell culture reagents were purchased from Gibco (Grand Island, NY, USA) or HyClone (Logan, UT, USA).

\subsection{Identification of Intracellular Localization}

DiI (1,1'-Dioctadecyl-3,3,3',3'-tetramethylindocarbocyanine perchlorate; Thermo Fisher, Waltham, MA, USA) was used as a lipophilic fluorescent probe of E, and miR-125 was labeled with FAM (carboxyl fluorescein; GenePharma). SAS cells were seeded overnight and incubated with DiI//LPN-KL or 
FAM-miR/SLN-KL for the indicated time. These cells were then fixed with $4 \%$ paraformaldehyde for $10 \mathrm{~min}$ and stained with $4^{\prime}$,6-diamidino-2-phenylindole (DAPI; blue) at $37^{\circ} \mathrm{C}$ to identify the nucleus. For DiI//LPN-KL intracellular localization, MitoTracker ${ }^{\circledR}$ Green (MitoGreen), LysoTracker ${ }^{\circledR}$ Green (LysoGreen), and an antibody against EGFR were added to monitor mitochondrial, lysosomal, and EGFR distribution. For the intracellular distribution of FAM-miR/SLN-KL (green), MitoTracker ${ }^{\circledR}$ Red (MitoRed; Thermo Fisher), LysoTracker ${ }^{\circledR}$ Red (LysoRed; Thermo Fisher), and an antibody against EGFR were used to detect mitochondrial, lysosomal, and EGFR localization. For both nanoparticle formulations, early endosomes were identified by immunofluorescence staining with an antibody against early endosome antigen 1 (EEA1) overnight. Images were obtained using a confocal laser scanning microscope (CLSM; Olympus FV10i; Olympus, Tokyo, Japan) with a 60× objective lens at a magnification of $1500 \times$. The representative images are shown $(n=3)$.

\subsection{Detection of Mitochondrial ROS Level Using Mito-SOX}

MitoSOX (Thermo Fisher) was used to monitor mitochondrial reactive oxygen species (ROS), especially superoxide. After treatment, $5 \times 10^{5}$ cells were mixed with $5 \mu \mathrm{M}$ of MitoSOX reagent at $37^{\circ} \mathrm{C}$ in the dark for $20 \mathrm{~min}$. After harvest, the fluorescence intensity of MitoSOX was detected using a FACSCalibur flow cytometer (BD Biosciences, San Jose, CA, USA) with excitation at $510 \mathrm{~nm}$ and emission at $580 \mathrm{~nm}$ [32]. The percentage change of mitochondrial ROS level by the treatment group is expressed relative to that of the control (CTR) without treatment (only medium) group.

\subsection{Measurement of Total Cellular ATP}

After treatment, the ATPlite luminescence One-Step ATP detection assay (Perkin Elmer, Waltham, MA, USA) was used to monitor ATP levels, based on firefly luciferase/luciferin activity measurement at $560 \mathrm{~nm}$ using a TECAN Infinite 200 multimode microplate reader (Tecan Inc., Mannedorf, Switzerland) [33].

\subsection{Measurements of Mitochondrial Respiration}

Extracellular acidification rate (ECAR) and the oxygen consumption rate (OCR) were evaluated using the Seahorse XF Glycolysis Stress Test Kit and Seahorse XF Cell Mito Stress Test Kit (Agilent Technologies, Palo Alto, CA, USA), respectively. After pretreatment with oligomycin (ATP synthase inhibitor; $1 \mu \mathrm{M}$ ), different formulations of $\mathrm{E}$ and/or miR-125-loaded formulations were treated, followed by injection of carbonylcyanide p-trifluoromethoxyphenylhydrazone (FCCP; protonophoric uncoupler; $1 \mu \mathrm{M}$ ) and antimycin A (electron transport chain inhibitor; $1 \mu \mathrm{M}$ ). OCR and ECAR were detected using a Seahorse XFe24 Extracellular Flux Analyzer (Seahorse Biosciences, North Billerica, MA, USA).

\subsection{Glucose Uptake Assay}

After treatment for the indicated time, cells were incubated with fluorescent D-glucose analog (2-[N-(7-nitrobenz-2-oxa-1,3-diazol-4-yl) amino]-2-deoxyglucose, 2-NBDG) for $10 \mathrm{~min}$ in the dark, washed by PBS, and then added with cell lysis buffer to prepare the cell extracts. These cell extracts were homogenized and centrifuged. The supernatants were transferred into 96-well, and the fluorescence was measured using a TECAN reader with excitation and emission at 485 and $535 \mathrm{~nm}$, respectively.

\subsection{Oil Red O Staining for Intracellular Lipid Accumulation}

After treatment, the cells were stained with Oil Red O (Sigma-Aldrich). The cells were washed twice with PBS, fixed with paraformaldehyde in PBS at room temperature for $1 \mathrm{~h}$. The cells were then washed with deionized water, stained with Oil Red $\mathrm{O}$ for $1 \mathrm{~h}$ in the dark. The stained lipid droplets were determined by measuring the absorbance at $492 \mathrm{~nm}$ using a TECAN reader. The percentage change of lipid accumulation by the treatment group is expressed relative to that of the CTR group [34]. 


\subsection{Evaluation of Protein Expression Levels via Western Blot}

After treatment, cells were lysed in an ice-cold lysis solution containing proteinase inhibitor to reduce protein degradation. The protein concentrations of supernatants were measured by BCA protein assay. Protein samples were run by SDS-polyacrylamide gel electrophoresis and then transferred onto polyvinylidene difluoride membranes. The blots were blocked in 5\% nonfat milk for $1 \mathrm{~h}$ and then incubated at $4{ }^{\circ} \mathrm{C}$ with primary antibodies against different proteins from Cell Signaling or Abcam (Supplementary Table S1). The samples were then added with horseradish peroxidase-conjugated secondary IgG. These blots were developed by enhanced chemiluminescence kits (PerkinElmer) and visualized with a Millipore detection system.

\subsection{Migration Assay}

After overnight seeding of SAS cells in inserts (Ibidi GmbH, Munich, Germany), a sterile pipette tip was used to make a scratch in each well of cancer cells. The cells were then treated with the different formulations and monitored for $15 \mathrm{~h}$. Images were photographed at the same site under a light microscope, and the migration area was calculated using Image J. Relative migration area\% was computed according to the following equation:

Relative migration area $(\%$ of area at $0 \mathrm{~h})=100 \%-[$ control area $(15 \mathrm{~h}) /$ control area $(0 \mathrm{~h}) \times 100 \%]$

\subsection{Cytotoxicity by Sulforhodamine B (SRB) Assay}

After overnight seeding of cells, various formulations of E with or without miR-125/SLN-KL were added to SAS or NOK cells incubated in 96-well plates at $37^{\circ} \mathrm{C}$ for $48 \mathrm{~h}$. The cytotoxic effects were determined by SRB assay. The cells were stained with $0.04 \%$ SRB for $10 \mathrm{~min}$ and washed with $1 \%$ acetic acid. Finally, the absorbance at $540 \mathrm{~nm}$ was monitored using a TECAN reader.

\subsection{Apoptosis Detection Assay}

After treatment, staining on the cells was performed with Annexin V-FITC/propidium iodide (PI) laboring solution for $15 \mathrm{~min}$ in the dark. Cell population \% in apoptosis and necrosis was detected and analyzed by a BD flow cytometer.

\subsection{Establishment of In Vivo SAS-Tumor Bearing Mouse Model}

BALB/c nude mice (6 weeks old, $22 \mathrm{~g}$ body weight) were obtained from the National Laboratory Animal Center and maintained in the Animal Center of National Yang-Ming University. All mice were kept on a half-day light-dark cycle. Animal care and handling procedures obey the guidelines and have been approved by the Institutional Animal Care and Use Committee. SAS cells were administered subcutaneously into the right cheek region of the male mice. Tumor size was calculated according to the following equation:

$$
\mathrm{V}=\left(\mathrm{L} \times \mathrm{W}^{2}\right) / 2
$$

where $\mathrm{L}$ (length, $\mathrm{mm}$ ) is the longest diameter, and $\mathrm{W}$ (width, $\mathrm{mm}$ ) is the shortest diameter perpendicular to the longest axis.

\subsection{Evaluation of Antitumor Efficacy on SAS Tumor-Bearing Mice}

The tumor size of mice was grown up to about $60 \mathrm{~mm}^{3}$ before treatment. SAS tumor-bearing mice were randomly divided into five groups $(n=5)$, namely, saline (control; CTR), E ( $80 \mathrm{mg} / \mathrm{kg}), \mathrm{E} / \mathrm{LPN}$, E/LPN-KL, and E/LPN-KL +miR125/SLN-KL (E, $80 \mathrm{mg} / \mathrm{kg}$; miR-125, $1.25 \mathrm{mg} / \mathrm{kg}$ ). Each group received different formulations every 2 days for 20 days. The tumor size of the mice was measured with a digital caliper every 2 days, and the tumor volume (V) was computed according to Equation (3). 


\subsection{Biochemical Tests}

Two days after the final treatment, the whole blood of $150 \mu \mathrm{L}$ was taken from the orbital sinus of mice. These blood samples were centrifuged to isolate the serum. The serum concentrations of glucose, cholesterol, glutamate pyruvate transaminase (GPT), creatinine (CRE), and creatine kinase-myocardial band (CKMB) were measured using a dry chemistry analyzer (FUJI DRI-CHEM 7000V, FUJIFILM Corporation, Tokyo, Japan) [35].

\subsection{Statistical Analysis}

Data are presented as the means \pm standard deviation. Student's $t$-test was used to analyze differences between the two treatment groups. A significant difference was set at $p<0.05$.

\section{Results}

\subsection{Physicochemical Characteristics of E- or miR-125-Loaded Nanoparticles}

The physicochemical characteristics of E/LPN-KL and miR-125/SLN-KL are shown in Figure 1. The size, zeta potential, polydispersity index (PdI), and encapsulation efficiency (EE\%) of these E- or miR-125-loaded nanoparticles are presented in Table 1. The schematic of miR125/SLN-KL or E/LPN-KL is displayed in Figure 1a. E/LPN-KL was spherical and had a homogeneous polymer core and a lipid shell modified with peptide K and L (Figure 1b). Similarly, miR125/SLN-KL also displayed spherical shape and had a homogeneous matrix and a surfactant/lipid shell coated with peptide K and L (Figure 1c).

The size, zeta potential, PdI, and encapsulation efficiency (EE\%) of these E- or miR-125-loaded nanoparticles are demonstrated in Figure $1 \mathrm{~d}-\mathrm{g}$ and Table 1 . The zeta potential of E/LPN-KL was around $-19 \mathrm{mV}$, indicating a negative charge due to the PLGA core $(n=3$; Table 1 , Figure $1 \mathrm{e})$. However, miR125/SLN-KL possessed a positive zeta potential of about $46 \mathrm{mV}$ ( $n=3$; Table 1, Figure 1g), primarily because of the positive charges of cationic lipids and peptides K and L. Interestingly, miR-125 might also be distributed on the surface of the nanoshell, possibly because of the electrostatic interaction between anionic miR and cationic lipids/peptides (Figure 1a-g).
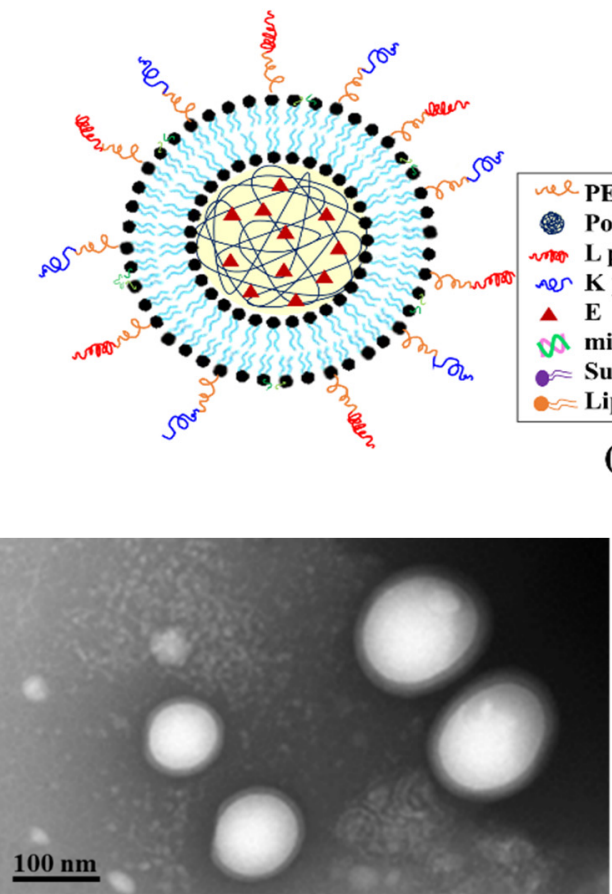

(b)

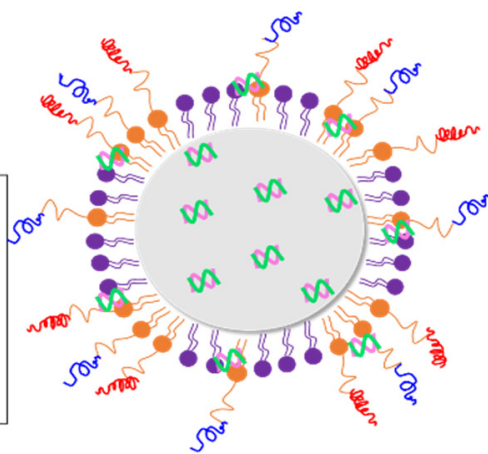

(a)

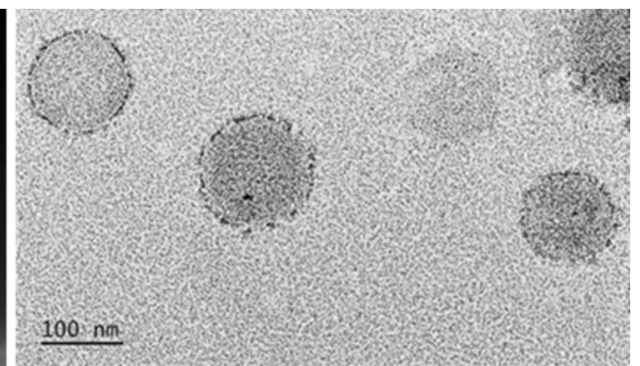

(c)

Figure 1. Cont. 


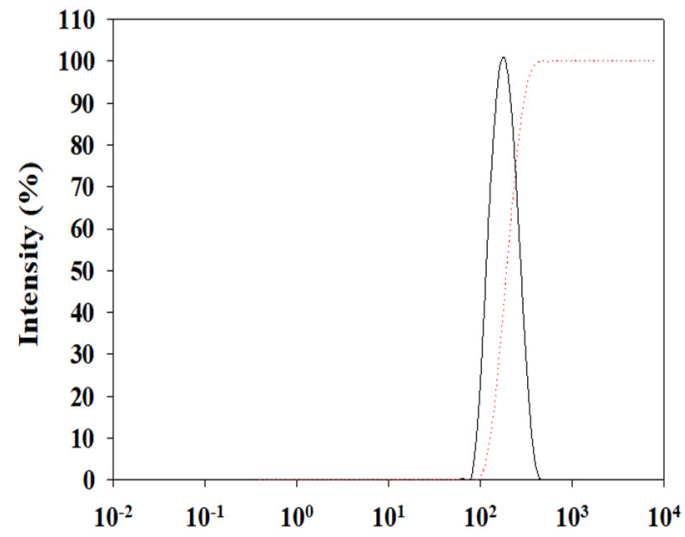

(d) Size (nm)

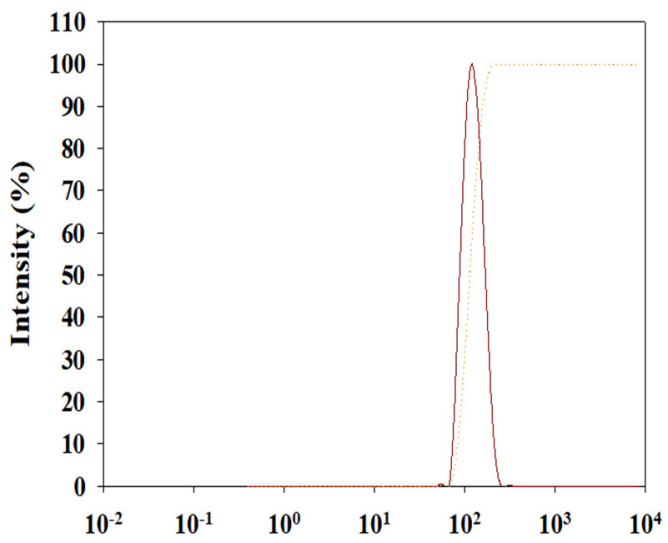

(f) Diameter (nm)

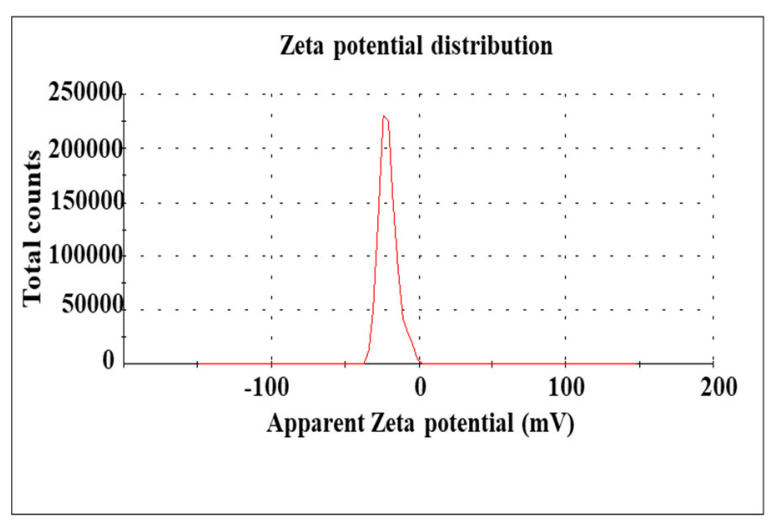

(e)

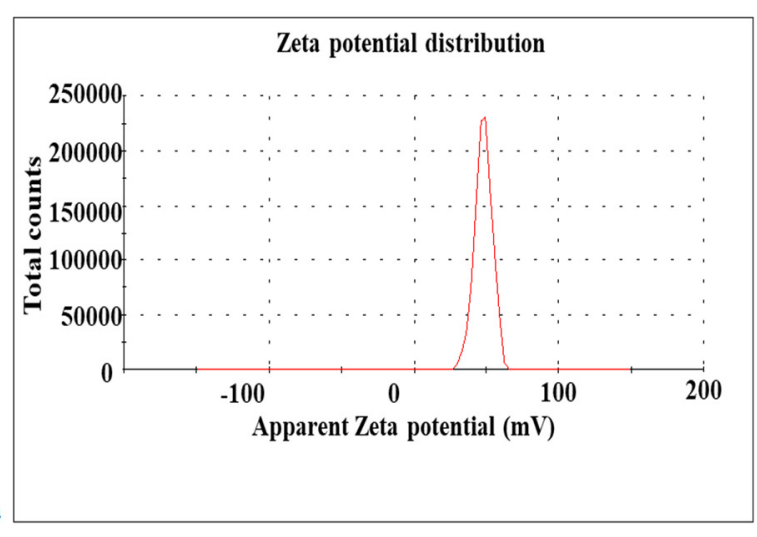

(g)

Figure 1. Schematic, morphological characteristics, particle size, and zeta potential of E/LPN-KL and miR-125/SLN-KL formulations. (a) A schematic of E/LPN-KL and miR-125/SLN-KL, (b,c) TEM images of (b) E/LPN-KL and (c) miR-125/SLN-KL, (d) size distribution and (e) zeta potential of E/LPN-KL, and (f) size distribution and (g) zeta potential of miR-125/SLN-KL. The representative plots are shown $(n=3)$. E, ellagic acid; LPN, lipid-polymer nanoparticle; SLN, solid lipid nanoparticles.

Table 1. Characteristics of E/LPN-KL and miR-125/SLN-KL.

\begin{tabular}{ccccc}
\hline Formulation & Particle Size $(\mathbf{n m})$ & PdI $^{\mathbf{a}}$ & Zeta Potential $(\mathbf{m V})$ & EE $^{\mathbf{b}} \mathbf{( \% )}$ \\
\hline E/LPN-KL & $195.23 \pm 5.88$ & $0.24 \pm 0.08$ & $-18.73 \pm 2.29$ & $85.53 \pm 1.35$ \\
miR-125/SLN-KL & $158.67 \pm 3.69$ & $0.20 \pm 0.06$ & $46.47 \pm 1.22$ & $86.28 \pm 1.56$ \\
\hline
\end{tabular}

$\mathrm{PdI}^{\mathrm{a}}$ : polydispersity index; $\mathrm{EE}^{\mathrm{b}}$ : encapsulation efficiency.

\subsection{Cellular Internalization of DiI/LPN-KL and FAM-miR125/SLN-KL into SAS Cells}

The intracellular localization of DiI/LPN-KL and FAM-miR125/SLN-KL in SAS cells was detected via CLSM, as shown in Figure 2. After treatment of DiI/LPN-KL (the molar ratio of DiI: lipid = 1: 10) for the indicated time, the cells were stained with DAPI (blue), MitoTracker Green (MitoGreen; green), and anti-EGFR antibody (gray) to localize the nucleus, mitochondria, and EGFR, respectively (Figure 2a). The intracellular localization of DiI/LPN-KL in SAS cells was visualized after 1, 10, and 30 min of incubation. After these three treatment periods, red fluorescence of DiI appeared mainly in the cytoplasm and mitochondria (Figure 2a). Mitochondrial targeting of DiI was the most predominant after $30 \mathrm{~min}$ of incubation (Figure 2a; bottom panels). A similar cellular distribution was also observed in FAM-miR/SLN-KL (Figure 2b). After the treatment of FAM-miR125 (100 nM)/SLN-KL for the 
specified time, the cells were stained with DAPI (blue), MitoRed (red), and anti-EGFR antibody (gray) to indicate the nucleus, mitochondria, and EGFR, respectively (Figure 2b). EGFR targeting of green FAM-miR/SLN-KL was observed after 10 and $30 \mathrm{~min}$. However, the cytoplasmic and mitochondrial localizations of FAM-miR were noticeable after $3 \mathrm{~h}$ of incubation (Figure $2 \mathrm{~b}$; bottom panels).

Endosomal escape is critical for the efficacious transport of nanoparticles to subcellular targets [36]. The cellular uptake of DiI/LPN-KL (red) was detected after $1 \mathrm{~min}, 30 \mathrm{~min}$, and $3 \mathrm{~h}$ of incubation (Figure 2c). The cells were then stained with DAPI (blue), LysoGreen (green), and anti-EEA1 antibody (gray) to localize the nucleus, lysosome, and endosome, respectively. Few red dots of DiI were observed in the endosomes at $1 \mathrm{~min}$ (Figure 2c; up panels), and the co-localization with lysosomes (yellow) was detected at 30 min (Figure 2c; middle panels). Furthermore, the red fluorescence of DiI was not co-localized with the green fluorescence of lysosomes, indicating that DiI was transported into the cytoplasm after delivery in SAS cells for $3 \mathrm{~h}$ (Figure 2c; bottom panels). A similar cellular distribution was also found in FAM-miR/SLN-KL. Partial endosomal and lysosomal entrapment of green FAM-miR was detected after 1 and $3 \mathrm{~h}$ (Figure 2d; up and middle panels). However, the cytoplasmic localization of FAM-miR was obvious after $8 \mathrm{~h}$ of incubation (Figure $2 \mathrm{~d}$; bottom panels).

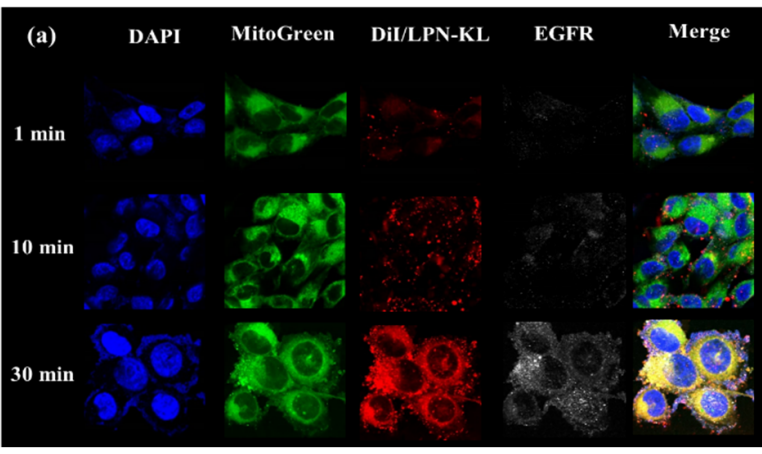

(a)

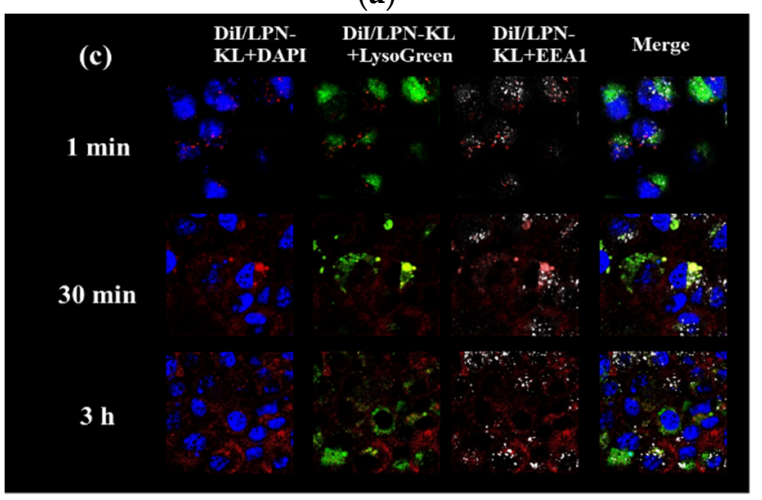

(c)

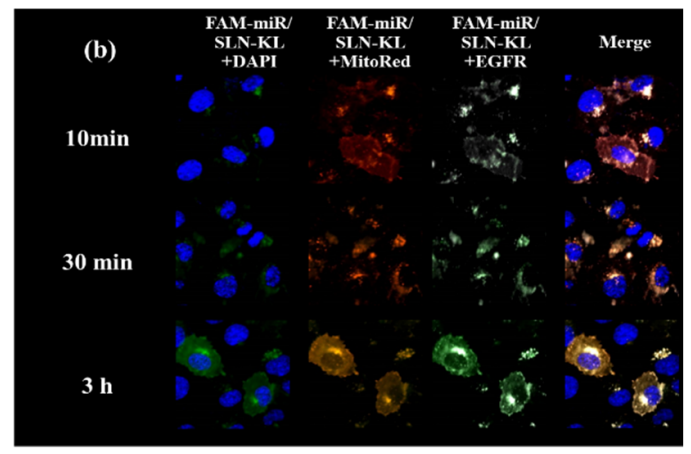

(b)

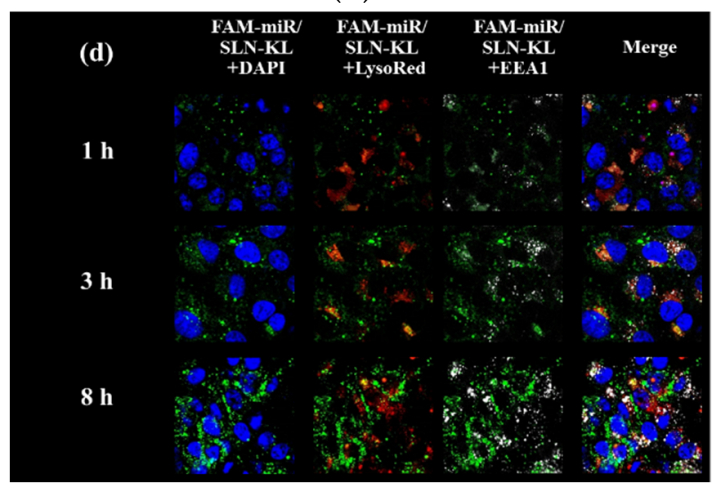

(d)

Figure 2. Cellular internalization and uptake of DiI or FAM-miR125/SLN-KL into human tongue squamous carcinoma SAS cells. (a,b) SAS cells were treated with $(\mathbf{a}-\mathbf{c})$ DiI in LPN-KL or $(\mathbf{b}-\mathbf{d})$ FAM-miR125/SLN-KL for the indicated time intervals and observed with CLSM. (a) Green, MitoGreen; red, DiI; blue, DAPI; gray, EGFR. (b) Green, FAM-miR125; red, MitoRed; blue, DAPI; gray, EGFR. (c) Green, LysoGreen; red, DiI; blue, DAPI; gray, EEA1. (d) Green, FAM-miR125; red, LysoRed; blue, DAPI; gray, EEA1. Magnification: 1500×. The representative images are shown $(n=3)$. DiI, 1,1'-Dioctadecyl-3,3,3', 3' -tetramethylindocarbocyanine perchlorate; FAM, carboxyl fluorescein; EEA1, early endosome antigen 1 .

\subsection{Effect of Different Treatments on Mitochondrial ROS Production and Bioenergenesis in SAS Cells}

The imbalance in mitochondrial oxidative metabolism may cause an increase in ROS levels, subsequently inducing oxidative stress and ultimately triggering the disruption in mitochondrial membrane potential (MMP; $\Delta \Psi_{\mathrm{m}}$ ) and apoptosis in various cancer cells $[37,38]$. In our study, after the 
SAS cells were treated with E, E/LPN, or E/LPN-KL, the relative mitochondrial ROS percentages, as indicated by the Mito-SOX fluorescence, were significantly higher than that of the control (Figure 3a). This mitochondrial ROS\% was the highest among all the formulations after the cells were incubated with E/LPN-KL+miR-125/SLN-KL ( $p<0.05$; Figure 3a). By contrast, $\Delta \Psi_{\mathrm{m}}$ or ATP levels after the treatment with E, E/LPN, or E/LPN-KL were considerably lower than those of the control (Figure 3b,c). Furthermore, E/LPN-KL plus miR-125/SLN-KL generated the highest degree of decrease in $\Delta \Psi_{\mathrm{m}}$ or ATP levels among all the formulations ( $p<0.05$; Figure $3 b, c)$.

Moreover, oligomycin (the ATP synthase inhibitor), FCCP (uncoupler), and antimycin (electron transport chain inhibitor) were used to evaluate mitochondrial function [39]. We found that the co-treatment of E/LPN-KL and miR125/SLN-KL diminished mitochondrial respiration and aerobic glycolysis to reduce the energy supply in SAS cells, as measured by a seahorse bioenergetics analyzer (Figure 3d,e). Previous works have emphasized that the change in cellular metabolic activities may modulate tumor initiation and progression [4,40]. Here, we demonstrated that the overall OCR and ECAR were considerably repressed by miR125/SLN-KL, E, E/LPN, and E/LPN-KL treatments to varying degrees compared with those found in the control group (Figure 3d,e). This result suggested the inhibitory effect of these formulations on aerobic glycolysis in SAS cells. The most remarkable reduction was observed in the miR125/SLN-KL+E/LPN-KL group (Figure 3d,e).

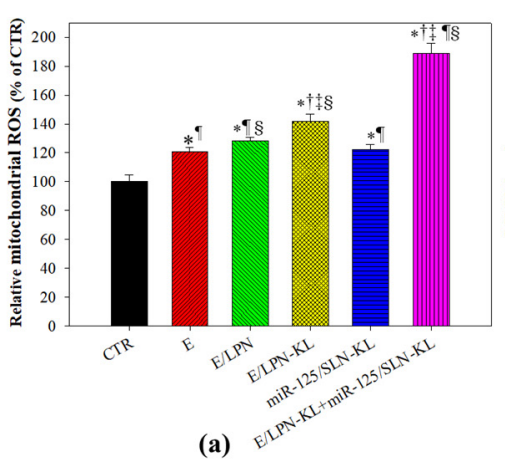

(a)

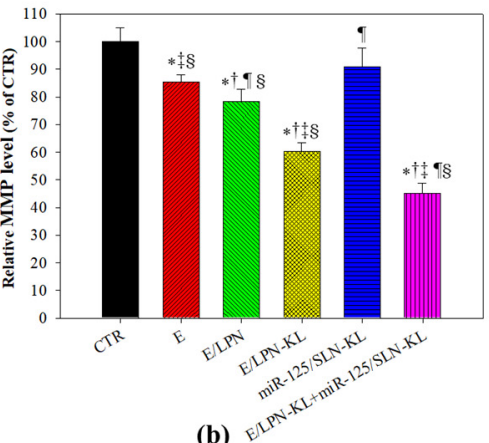

(b)

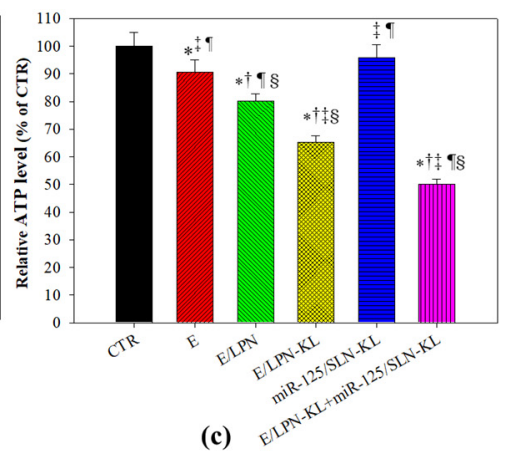

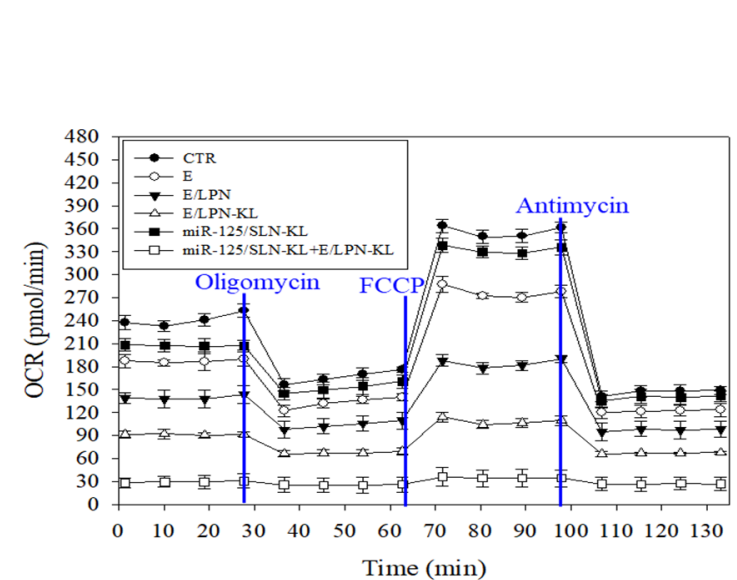

(d)

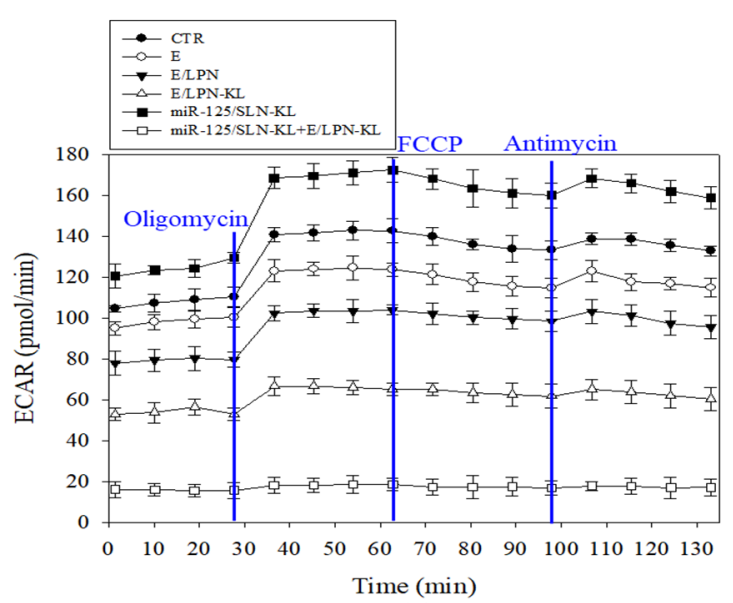

(e)

Figure 3. Effect of different treatments on mitochondrial reactive oxygen species (ROS), bioenergenesis, and dynamics in SAS cells. (a) Relative ROS percentage. (b) Relative ATP percentage. (c) Relative mitochondrial membrane potential (MMP) percentage. (a-c) Control (CTR) was normalized as 100\%. The mean fluorescence intensity of the other treatments was normalized relative to CTR. Data are presented as means $\pm \mathrm{SD}$ from $n=3 .{ }^{*} p<0.05$ compared with $\mathrm{CTR},{ }^{\dagger} p<0.05$ compared with E, $\ddagger p<0.05$ compared with E/LPN, ${ }^{\mathbb{I}} p<0.05$ compared with E/LPN-KL, and ${ }^{\S} p<0.05$ compared with miR-125/SLN-KL. (d) Oxygen consumption rate (OCR) and (e) extracellular acidification rate (ECAR) related to mitochondrial dynamics in SAS cells. 


\subsection{Increase in Glucose Uptake in SAS Cells Treated with Different Nanoparticle Formulations}

When SAS cells were treated with E, E/LPN, E/LPN-KL, miR-125/SLN-KL, and E/LPN-KL+miR-125/ SLN-KL, the glucose uptake into cells was significantly elevated compared to the control group in SAS cells (Figure 4a). Furthermore, when SAS cells were treated with E/LPN-KL+miR-125/SLN-KL, the cells showed the most significant increase in the glucose uptake compared to the control group (Figure 4a).

\subsection{Decreased Accumulation of Oil Droplets in SAS Cells Treated with Different Nanoparticle Formulations}

When the cells were treated with E and miR-125-loaded formulations, the accumulation of oil droplets in the cells significantly decreased compared with that in the control group, especially in the treatment group of E/LPN-KL (Figure $4 b$ ). Furthermore, the cells co-treated with E/LPN-KL and miR-125/SLN-KL exhibited the most remarkable reduction in the lipid droplet accumulation among all the groups (Figure $4 b$ ).

\subsection{Evaluation of Proteins Associated with Adipogenesis and Lipid Synthesis by Western Blot}

Figure $4 \mathrm{c}$ illustrates that E-containing formulations downregulated adipogenic factors, such as preadipocyte factor 1 (Pref1), peroxisome proliferator-activated receptor-gamma (PPAR $\gamma$ ), PPAR $\gamma$ coactivator $1 \alpha(\mathrm{PGC} 1 \alpha)$, the transcription factor cAMP response element-binding protein $\alpha(\mathrm{C} / \mathrm{EBP} \alpha)$, and adipocyte protein 2 (aP2), to varying degrees at the protein level. Strikingly, the combined treatment of E/LPN-KL and miR125/SLN-KL most significantly suppressed the expression of these proteins associated with adipogenesis and lipid synthesis among all the treatment groups (Figure 4c). E-loaded formulations, particularly E/LPN-KL and its co-treatment with miR125/SLN-KL, inhibited the expression of PGC1B. Thus, thermogenesis might be suppressed in SAS cells (Figure 4c).

\subsection{Assessment of Proteins Related to Mitophagy and Necropotosis by Western Blot}

Interestingly, when SAS cells were treated with E- and miR125-loaded formulations, mitochondrial autophagy/mitophagy-related proteins were altered to various extents. The expression of PINK1/Parkin substantially increased, but the expression of tumor necrosis factor receptor associated protein 1 (TRAP1) decreased in response to the treatment of $\mathrm{E}$ and miR125, especially for the co-treatment of E/LPN-KL and miR125/SLN-KL (Figure 4d). Optic atrophy 1 (OPA1), a mitochondrial dynamin-like GTPase, was cleaved, whereas the phosphorylation of dynamin related protein (DRP) 1, a mitochondrial fission marker, was increased in response to the treatment of E/LPN-KL and/or miR125/SLN-KL (Figure 4d). Moreover, autophagy- and necropotosis-related proteins, including microtubule-associated protein 1 light chain 3 (LC3) II, Beclin 1, autophagy related 5 (Atg5), and RIP1/3, were upregulated after incubation with $\mathrm{E}$ and miR125, particularly for the combination therapy of E/LPN-KL and miR125/SLN-KL. 


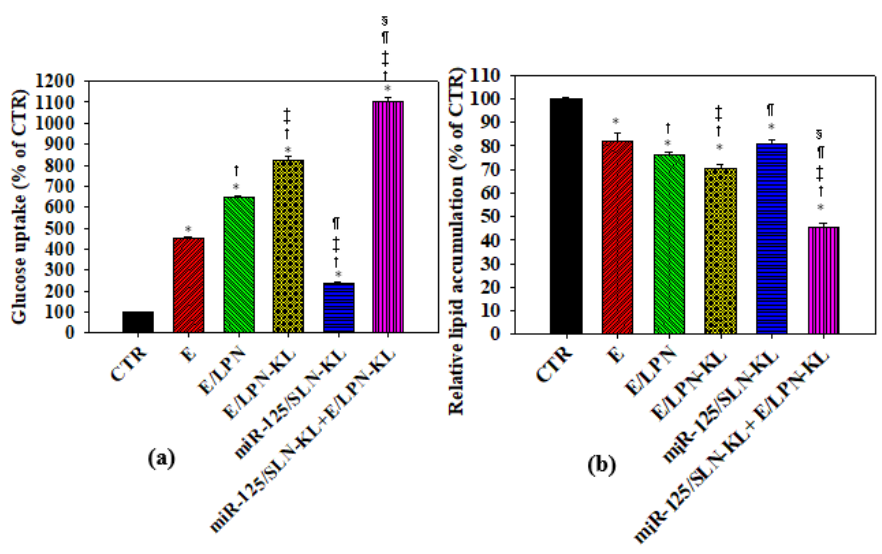

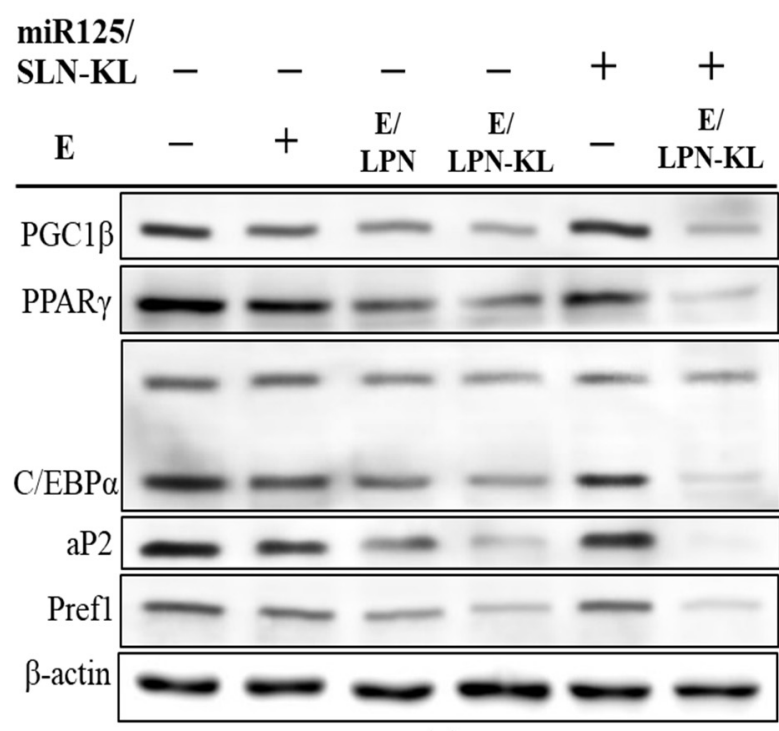

(c)

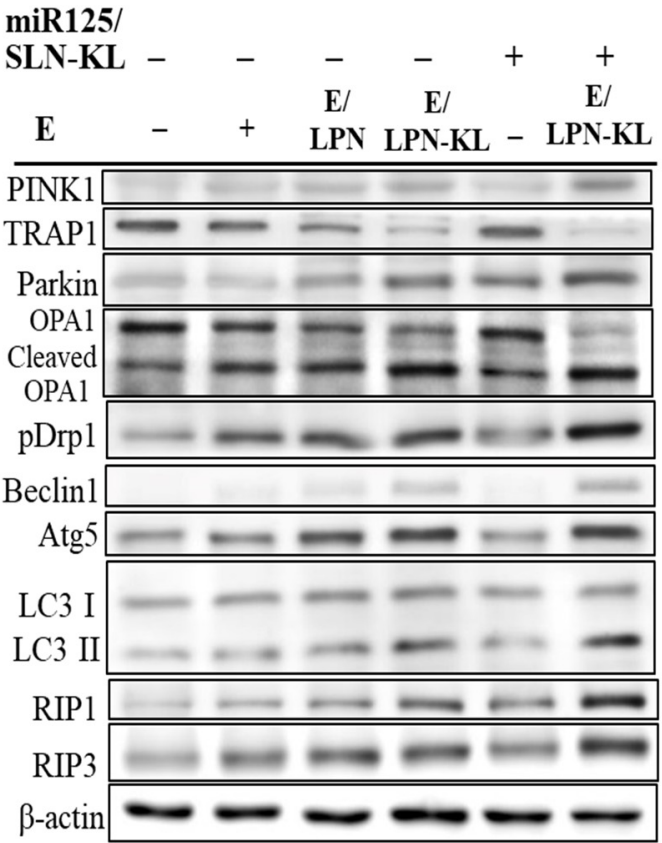

(d)

Figure 4. Effect of different formulations on the relative levels of (a) glucose uptake and (b) lipid accumulation. For $(\mathbf{a}, \mathbf{b}):{ }^{*} p<0.05$ : compared with CTR, ${ }^{\dagger} p<0.05$ compared with E/LPN, $\ddagger p<0.05$ compared with E/LPN, ${ }^{\mathbb{I}} p<0.05$ compared with E/LPN-KL, and ${ }^{\S} p<0.05$ compared with miR125/LPN-KL via Student's $t$-test analysis, respectively. (c,d) The expression of proteins associated with (c) lipid or glucose metabolism and (d) mitochondrial dynamics, fusion/fission, mitophagy, and necroptosis in SAS cells. After various treatments, the cells were evaluated with Western blot, and the representative blots were shown $(n=3)$.

\subsection{Reduced Migration of SAS Cells Treated with E-and miR-125-Loaded Formulations}

The result of the migration assay performed on Transwell inserts showed that the treatment with free E mildly decreased cell migration by approximately 30\% (Figure $5 \mathrm{a}, \mathrm{b}$ ). The migration area of SAS cells was further reduced by the treatment of E/LPN or E/LPN-KL (Figure 5a,b). Notably, the combined treatment of miR125/SLN-KL and E/LPN-KL further diminished the cell migration percentage to $28.94 \% \pm 2.96 \%$ (Figure $5 \mathrm{a}, \mathrm{b}$ ). The protein expression levels of $\beta$-catenin, EMT, and multidrug resistance (MDR) pathways in SAS cells were assessed via Western blot after these cells were treated with miR-125- and E-incorporated preparations. Lone miR-125- and E-loaded formulations attenuated the protein expression associated with $\beta$-catenin, EMT, and MDR pathways to varying degrees. Moreover, the co-modulation by miR125/SLN-KL and E/LPN-KL remarkably abolished the expression of $\beta$-catenin, 
$\mathrm{N}$-cadherin, vimentin, Snail, Slug, P-glycoprotein (P-gp), MDR-associated protein (MRP)1, and MRP2 and markedly intensified the E-cadherin expression compared with those in the other treatment groups (Figure 5c). These results indicated the superior suppressive effect of the co-treatment of miR125/SLN-KL and E/LPN-KL on the metastasis and resistance of SAS cells.

We further evaluated the possible cellular uptake enhancement of $\mathrm{E}$ in different formulations into SAS cells. The intracellular intensity of $\mathrm{C} 6$, a green fluorescent marker of $\mathrm{E}$, after being transported by various LPN formulations, was measured using a flow cytometer. In Figure $5 \mathrm{~d}$, a significant difference was observed when C6 was delivered by LPN or LPN-KL $(p<0.05)$. After the combined treatment with miR125/SLN-KL, the cellular intensity of C6/LPN-KL remarkably increased compared with that of the group without co-treatment. Interestingly, the group of miR125/SLN-KL+C6/LPN-KL exhibited the highest fluorescence intensity of $\mathrm{C} 6$ among the groups ( $p<0.05$; Figure $5 \mathrm{~d}$ ).

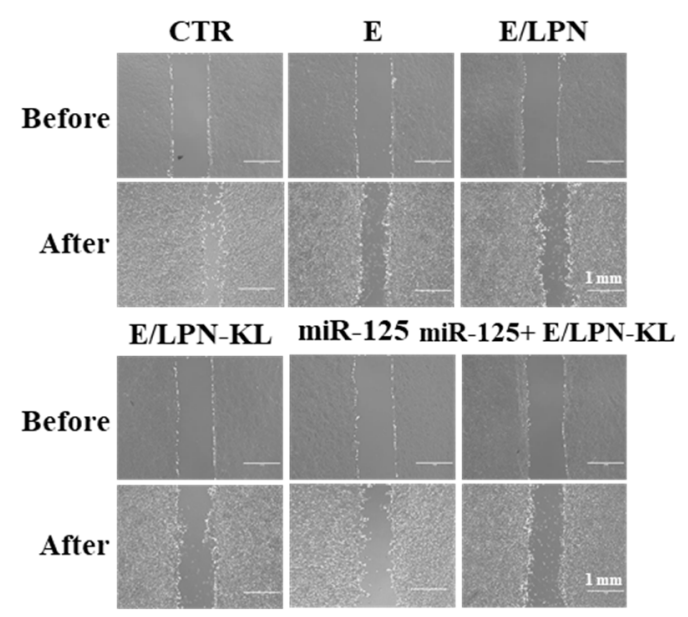

(a)

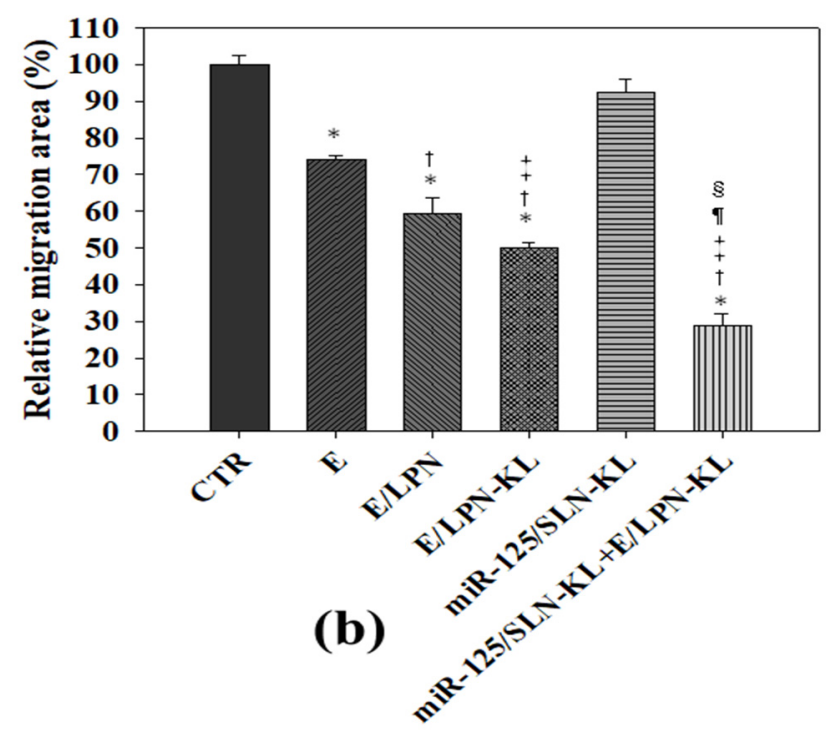

Figure 5. Cont. 


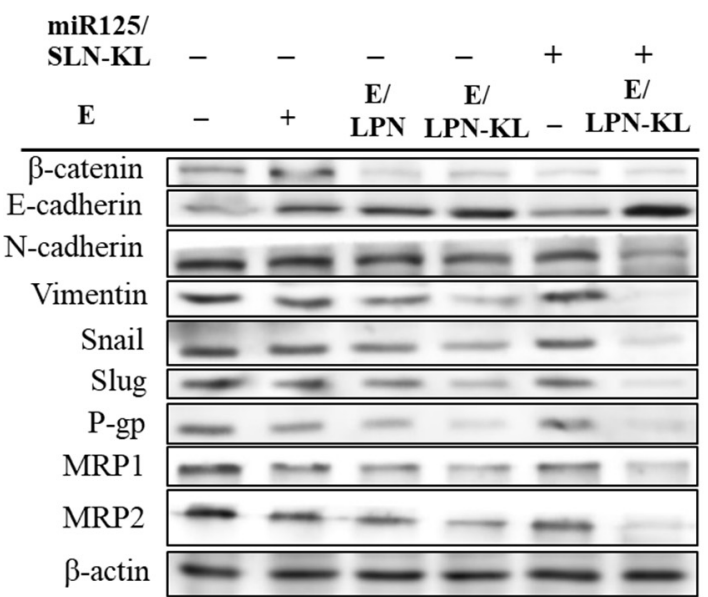

(c)

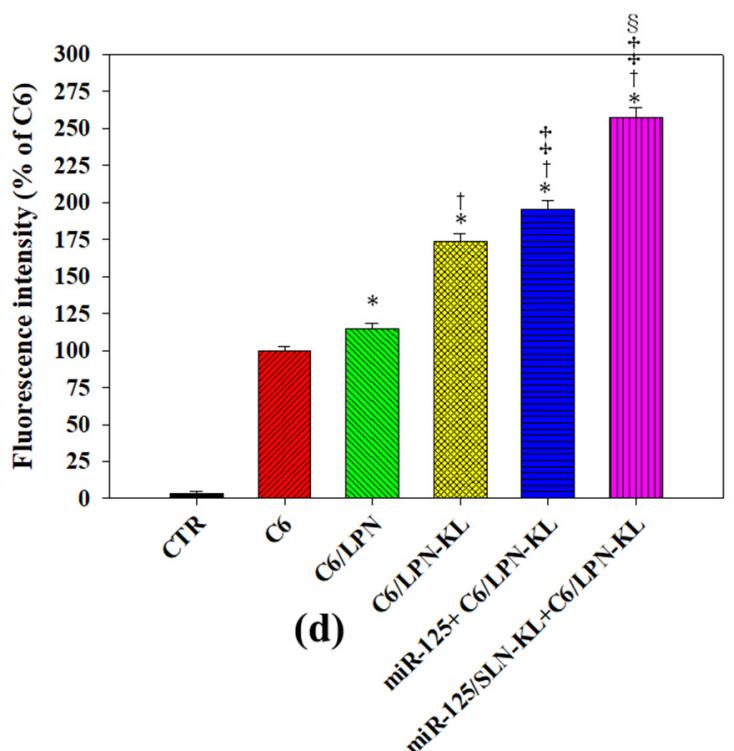

Figure 5. Effect of various treatments on migration and multidrug resistance (MDR) pathway in SAS cells. (a) The migration assay was conducted to evaluate the SAS cells treated with different formulations of E and miR-125 for $15 \mathrm{~h}$. Cellular images were then observed under a microscope. (b) Relative percentages of the cell migration area. ${ }^{*} p<0.05$ : compared with $\mathrm{CTR},{ }^{\dagger} p<0.05$ compared with $\mathrm{E} / \mathrm{LPN}, \ddagger p<0.05$ compared with $\mathrm{E} / \mathrm{LPN},{ }^{\mathbb{I}} p<0.05$ compared with $\mathrm{E} / \mathrm{LPN}-\mathrm{KL}$, and ${ }^{\S} p<0.05$ compared with miR125/LPN-KL via Student's $t$-test analysis. (c) The protein expression levels of epithelial-mesenchymal transition (EMT) and MDR were evaluated via Western blot after the SAS cells were given various treatments for $48 \mathrm{~h}$. The experiments were performed in triplicate with similar results. (d) The cellular uptake of coumarin-6 (C6; a probe of E) was monitored by detecting the relative green fluorescence intensity of $\mathrm{C} 6$ by using a flow cytometer. ${ }^{*} p<0.05$ compared with $\mathrm{C} 6$, ${ }^{\dagger} p<0.05$ compared with C6/LPN, ${ }^{\ddagger} p<0.05$ compared with C6/LPN-KL, and ${ }^{\S} p<0.05$ compared with miR125+C6/LPN-KL via Student's $t$-test analysis, respectively.

\subsection{Cytotoxicity of E in Various Formulations on NOK and SAS Cells}

NOK cells were used to evaluate the possible toxic effects of these miR-125- and E-loaded formulations on normal cells by using the SRB assay. We selected the E concentration of $20 \mu \mathrm{M}$, which was approximately IC30 (the concentration of 30\% inhibition of cell viability) of E on NOK cells and IC20 of E on SAS cells (Figure 6a,b). E was encapsulated in various formulations in the presence and absence of miR125/SLN-KL, and their cytotoxic effects on NOK cells after $48 \mathrm{~h}$ of incubation were compared with those of the control and E groups (Figure 6a). The result showed that the cell viability after the treatment with E/LPN or E/LPN-KL with or without miR125/SLN-KL increased to approximately $80-85 \%$ (Figure $6 \mathrm{a}$ ). Therefore, E/LPN-KL or its co-treatment with miR125/SLN-KL reduced the toxic effects of free $\mathrm{E}$ on NOK cells (Figure 6a).

We tested the effect of $\mathrm{E}$ and/or miR-125-loaded formulations on the viability of SAS cells. We found that $20 \mu \mathrm{M}$ E diminished the cell viability of SAS cells to $79.97 \%$. E/LPN-KL decreased the viability of SAS cells to $74.70 \%$ (relative percentage of the control group; Figure $6 \mathrm{~b}$ ). Interestingly, E/LPN-KL+miR-125/SLN-KL further reduced the viability of SAS cells to $57.88 \% \pm 0.67 \%$ (Figure $6 \mathrm{~b}$ ). Notably, E/LPN-KL+miR-125/SLN-KL displayed the highest inhibitory effect on SAS cell viability among all these formulations (Figure $6 \mathrm{~b}$ ). 

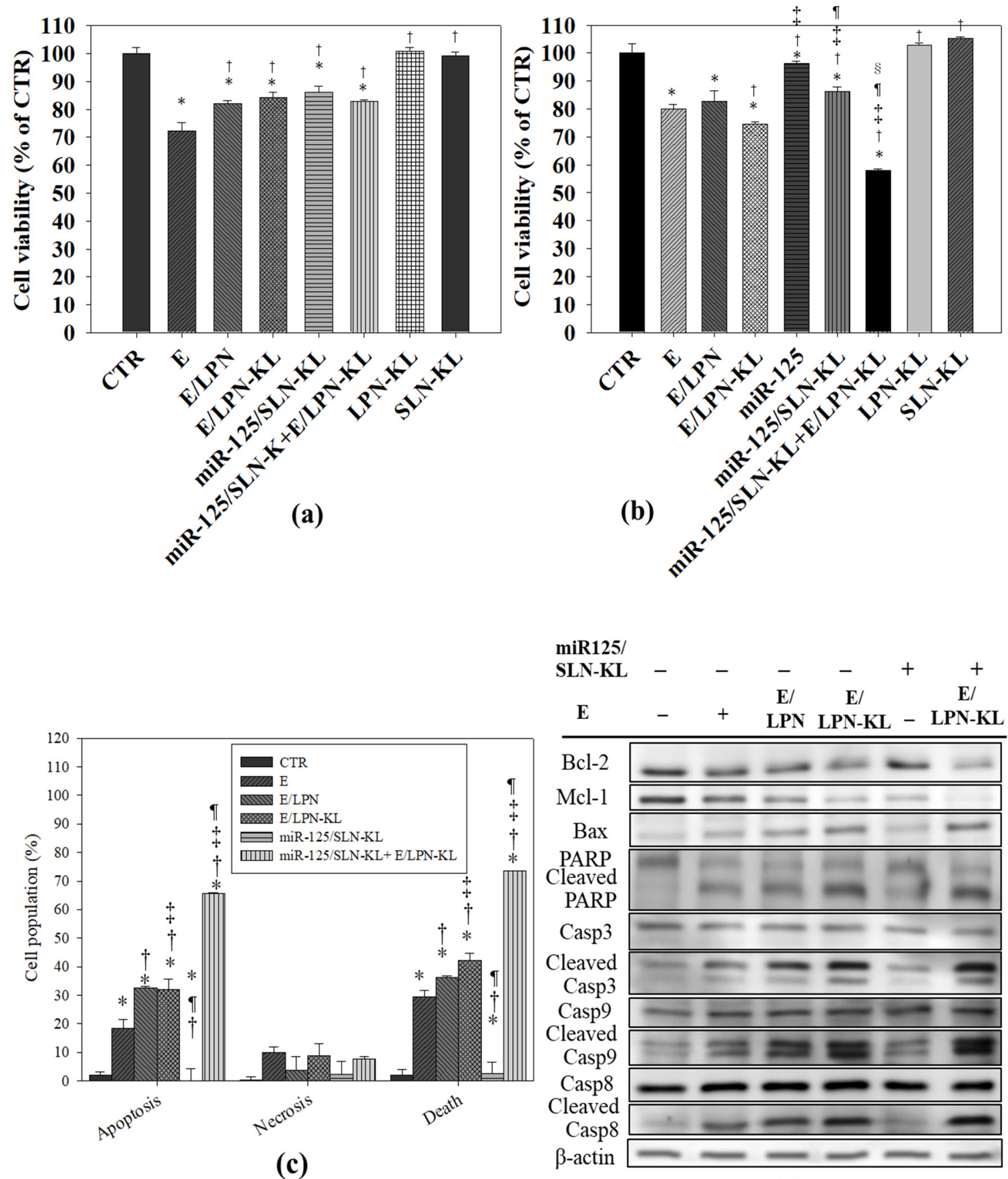

(d)

Figure 6. (a,b) Cytotoxicity of E and miR-125 in various formulations on normal and cancer cells. (a) NOK (normal oral keratinocyte) and (b) SAS cells were treated with E and/or miR-125 in various formulations for $48 \mathrm{~h}$. Cell viability was measured with sulforhodamine B (SRB) assay. Values are the mean $\pm \mathrm{SD}(n=3)$. For $(\mathbf{a}):{ }^{*} p<0.05$ compared with CTR; ${ }^{\dagger} p<0.05$ compared with E. For $(\mathbf{b}):{ }^{*} p<0.05$ compared with CTR; ${ }^{\dagger} p<0.05$ compared with E; ${ }^{\ddagger} p<0.05$ compared with E/LPN-KL; ${ }^{\mathbb{I}} p<0.05$ compared with miR-125; $\$ p<0.05$ compared with miR-125/SLN-KL. (c) The relative percentage of the apoptotic, necrotic, and dead cell population by Annexin V and propidium iodide (PI) assay. ${ }^{*} p<0.05$ compared with CTR; ${ }^{\dagger} p<0.05$ compared with E; ${ }^{\ddagger} p<0.05$ compared with E/LPN; ${ }^{\mathbb{I}} p<0.05$ compared with E/LPN-KL. (d) Effect of different treatments on the expression of proteins associated with the apoptosis of SAS cells. After various treatments, the cells were evaluated with Western blot, and their representative images were shown $(n=3)$. 


\subsection{Apoptotic Effect of E- and miR-125-Loaded Formulations on SAS Cells}

Cell apoptosis and necrosis (\%) were assessed via Annexin V-PI double staining on SAS cells. The result indicated that the apoptosis percentage (\% of the sum of early and late apoptotic cells) and death percentage ( $\%$ of the sum of apoptotic and necrotic cells; Figure $6 \mathrm{c}$ ) activated by E/LPN-KL were substantially higher than those triggered by E $(p<0.05)$. Noticeably, the number of apoptotic and dead cells due to the combined treatment of E/LPN-KL and miR125/SLN-KL was considerably more than that of the cells subjected to the other treatments (Figure 6c). Furthermore, marginal differences in the low percentage of the necrotic population were observed among all the formulations $(p>0.05$; Figure 6c).

The protein expression of the apoptosis-associated pathway of SAS cells after $48 \mathrm{~h}$ of treatments was assessed via Western blot. Myeloid cell leukemia 1 (Mcl-1) and Bcl-2, two anti-apoptotic proteins, were marginally inhibited by $\mathrm{E}$ but were significantly suppressed by the combined treatment of E/LPN-KL and miR125/SLN-KL (Figure 6d). On the contrary, the expression levels of pro-apoptotic proteins, such as Bax, cleaved poly (ADP-ribose) polymerase (PARP), caspase-3, caspase- 8 , and caspase-9, were slightly increased by the lone treatment of $\mathrm{E}$, but these apoptosis-inducing proteins were progressively increased by the treatments of E/LPN-KL and miR125/SLN-KL (Figure 6d).

\subsection{Serum Cholesterol and Glucose Levels In Vivo}

On the basis of our in vitro data, we further investigated the effect of E, E/LPN, E/LPN-KL, miR-125/SLN-KL, and E/LPN-KL+miR-125/SLN-KL formulations on blood cholesterol and glucose levels in mice (Figure $7 \mathrm{a}, \mathrm{b}$ ). When SAS-bearing mice were treated with various formulations, the cholesterol and glucose levels in all the treatment groups of E, E/LPN, E/LPN-KL, miR-125/SLN-KL, and E/LPN-KL+miR-125/SLN-KL were significantly reduced compared with those in the control group (Figure 7a,b). The most significant decrease in serum cholesterol and glucose levels was found in the combined group of E/LPN-KL and miR-125/SLN-KL (Figure 7a,b).

\subsection{In Vivo Biosafety Evaluation}

Serum CKMB, GPT, and CRE levels were monitored to examine the heart, liver, and renal functions, respectively (Figure 7c-e). Marginal or mild changes were observed in these three serum parameters after the treatment with E, E/LPN, or E/LPN-KL or its co-treatment with miR125/SLN-KL. Nevertheless, no substantial alterations were detected in these three parameters (Figure 7c-e). Interestingly, $\mathrm{E}$ has elicited a hepatoprotective effect against valproic acid-provoked hepatic injury in rats [41]. Our formulations did not cause serious organ toxicities and thus did not have obvious effects to alleviate the heart-, liver-, and kidney-related toxicities.

\subsection{In Vivo Antitumor Efficacy and Body Weight Studies on SAS Tumor-Bearing Mice}

The antitumor efficacy of various E- and miR-125-loaded formulations was detected in SAS tumor-bearing mice in vivo. The combined treatment of tumor-targeted E/LPN-KL and miR125/SLN-KL demonstrated the most noteworthy antitumor efficacy on SAS-bearing mice. This result suggested the greatest tumor-suppression ability of these combinatorial nanoparticle formulations among the different treatment groups (Figure 8a). For the safety evaluation, the body weight of SAS-bearing mice was also assessed. The untreated mice showed a mild decrease in body weight, possibly because of the increasing tumor burden (Figure 8b). All the other groups exhibited a constant increase in body weight and slight individual differences (Figure $8 b$ ), indicating the improvement in tumor burden-related weight loss. 


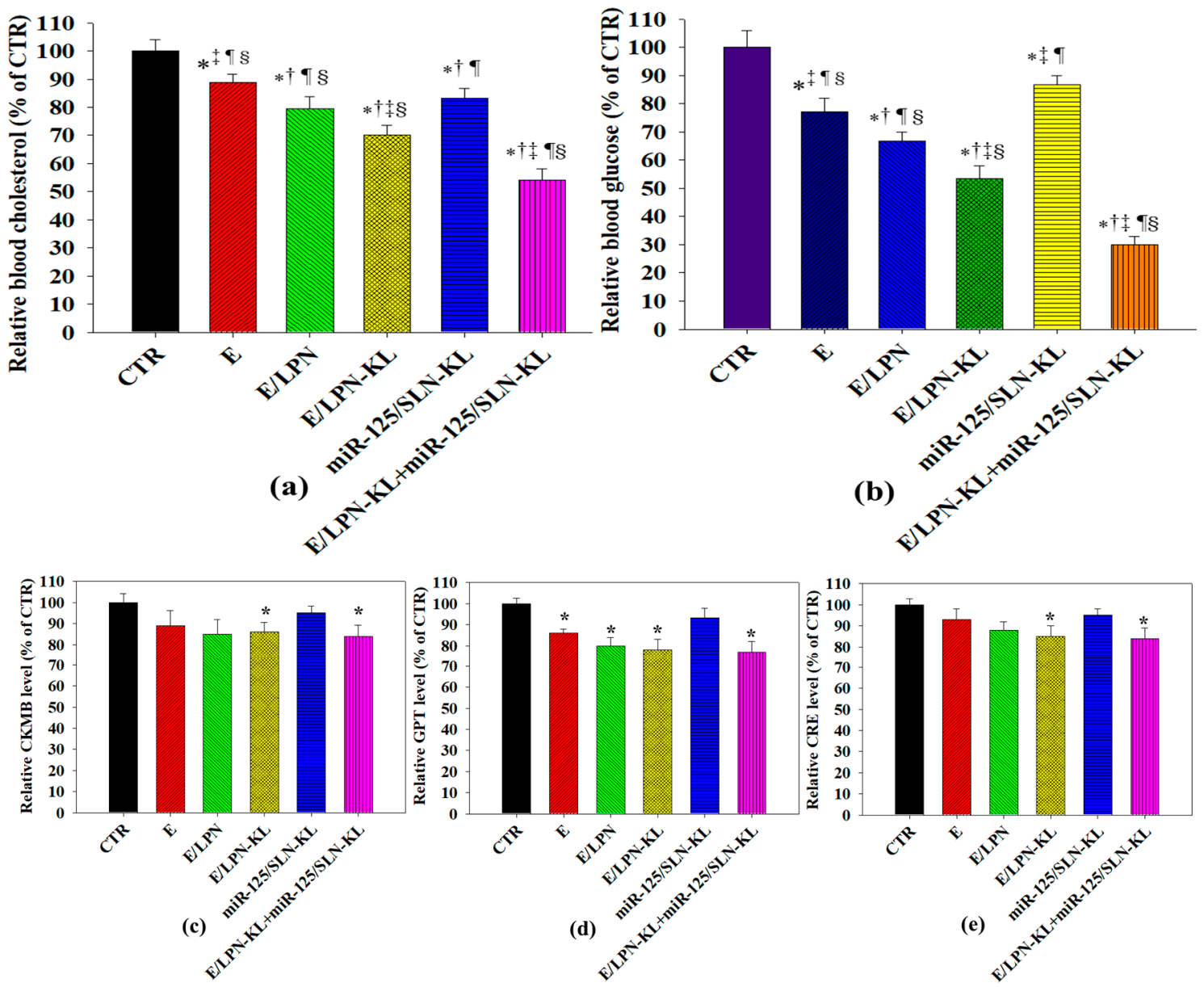

Figure 7. In vivo quantitation of blood (a) cholesterol, (b) glucose, (c) creatine kinase-myocardial band (CKMB), (d) glutamate pyruvate transaminase (GPT), and (e) creatinine (CRE) levels in SAS-bearing mice in different treatment groups. ${ }^{*} p<0.05$ compared with CTR, ${ }^{\dagger} p<0.05$ compared with E, $\ddagger p<0.05$ compared with E/LPN, II $p<0.05$ compared with E/LPN-KL, and ${ }^{\S} p<0.05$ compared with miR-125/SLN-KL.

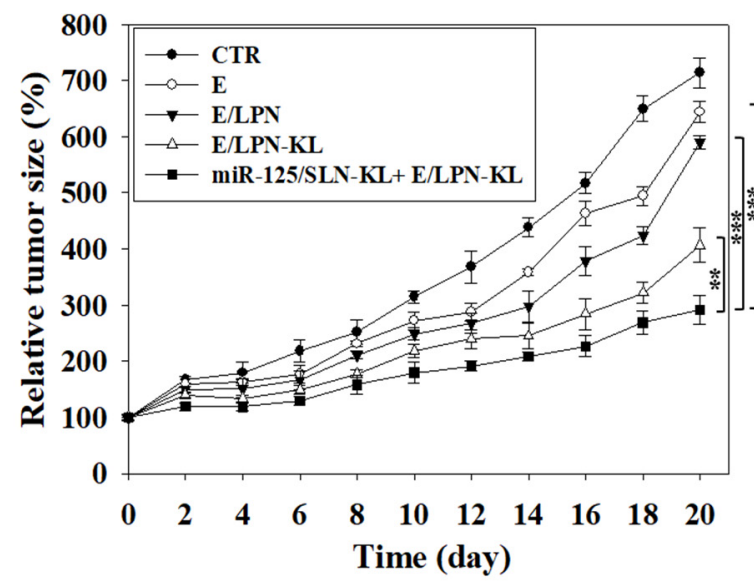

(a)

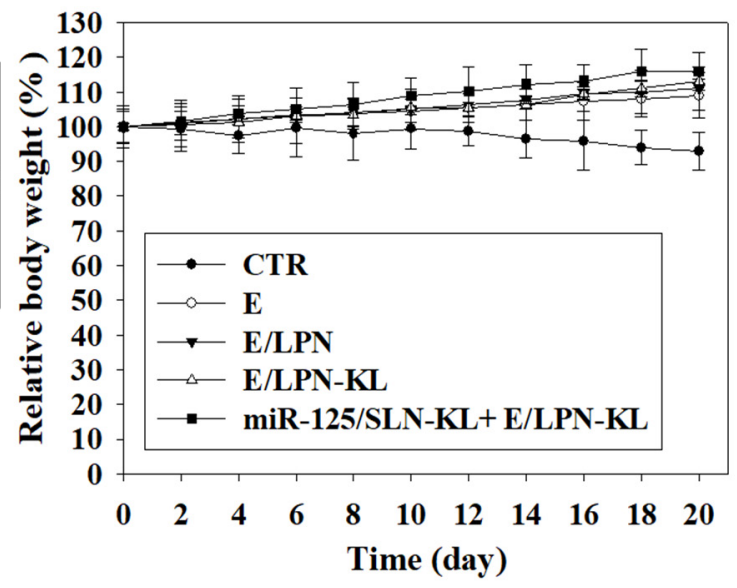

(b)

Figure 8. Antitumor efficacy and body weight studies on SAS-bearing mice treated with different formulations. (a) Antitumor efficacy of various formulations intravenously injected into SAS-bearing mice. Tumor growth was measured with digital calipers every 2 days (statistical significance: ${ }^{* *} p<0.01$; *** $p<0.001$.); (b) body weight as a function of time in CT-26-bearing mice. 


\section{Discussion}

The mitochondria of cancer cells usually have altered physiological processes, including hindered oxidative phosphorylation and mitochondrial hyperpolarization [4]. Furthermore, cancer cells demonstrate the atypical metabolism of the Warburg effect by displaying higher glycolysis and lactate production levels and misbalanced mitochondrial ATP generation [42]. These changes may affect the regulation of bioenergetics and the cellular oxidation-reduction (redox) equilibrium [3], leading to tumorigenesis, progression, angiogenesis, and chemotherapy resistance in cancer cells [43]. However, information about the regulation of these complicated factors of the mitochondrial bioenergetics on modulating HNC cell death is limited. Hence, in the present study, we aimed to evaluate the modulating effects of E/LPN-KL and miR125/SLN-KL on mitochondrial dynamics, energy metabolism, and anticancer efficacy in SAS cells.

$\mathrm{E}$ is abundant in oak, nuts, and fruits [14]. E in chestnut extract inhibits cell proliferation, induces apoptosis, modulates mitochondrial depolarization, and affects the cytokinomic and metabolomic properties of HepG2 cells [44]. Besides, miR-125 triggers pluripotent regulation in a wide variety of cancers, such as CRC, HNC, and hepatocellular carcinoma (HPC) [18-20]. Accordingly, the miR-125 mimic induces CRC cell apoptosis and reduces cell viability [19]. Furthermore, miR-125 upregulation in OSCC causes escalated oxidative stress and improves drug sensitivity against OSCC [45]. In particular, miR-125 transfection re-sensitizes HPC cells to 5-FU, possibly through the inhibition of glucose uptake and lactate production by directly targeting hexokinase II [46].

In this study, we used MitoTracker to verify the targeting delivery of $\mathrm{E}$ and miR-125 to the mitochondria via the formulation of LPN-KL or SLN-KL, respectively (Figure 2a,b). We found that the increased ROS generation (Figure 3a) and mitochondrial depolarization (Figure 3b) caused mitochondrial dysfunction in SAS cells (Figure 3c-e). The formulation of miR125/SLN-KL+E/LPN-KL most significantly disrupted mitochondrial respiration, aerobic glycolysis, and cellular bioenergetics, including OCR, ECAR, and ATP production (Figure 3c-e), indicating the excellent efficacy of this combinatorial therapy on modulating mitochondrial bioenergetics and dynamics in SAS cells.

Lipid production and accumulation are important for tumor progression because these processes may produce energy for tumorigenesis and provide the building components of phospholipids to form cell membranes [47]. PGC-1, a transcriptional coactivator, is the major regulator of mitochondrial bioenergenesis associated with energy metabolism by interacting with the nuclear receptor PPAR- $\gamma$ and C/EBP $\alpha[48,49]$. PGC- $1 \beta$ is pivotal to activate fatty acid $\beta$-oxidation [47]. Figure 4 shows that the co-treatment of E/LPN-KL and miR125/SLN-KL remarkably increased glucose uptake (Figure 4a) and significantly reduced lipid accumulation (Figure $4 \mathrm{~b}$ ) in SAS cells. The combinatorial therapy also considerably diminished the protein expression levels of adipogenic factors and metabolism of lipids, including Pref1, PGC1B, PPAR $\gamma, \mathrm{C} / \mathrm{EBP} \alpha$, and aP2, among all the treatment groups (Figure 4c). This result implied that this combined formulation might disturb lipid metabolism and mitochondrial bioenergenesis, thereby suppressing tumor progression. Figure $4 \mathrm{c}$ is consistent with the result of Woo et al. [50], who suggested that E might regulate adipogenesis at the early and middle stages to inhibit lipid synthesis [50]. Moreover, pomegranate E modulates lipid accumulation and cholesterol metabolism in human hepatic cells by regulating the PPAR $\gamma$-related pathway [51]. Additionally, the hepatic removal of PGC- $1 \beta$ modulates anabolic metabolism, thereby potentiating ROS-provoked damages of DNA, proteins, and lipids [52]. Consequently, the mitochondrial dynamic alteration induces cancer cell apoptosis and inhibits HPC tumor growth in mice [52].

The balance between two important housekeepers, namely, Parkin-mediated mitophagy and fusion/fission, is critical to maintaining mitochondrial homeostasis [4]. Mitophagy is provoked by numerous stimuli, such as oxidative stress and increased oxidative phosphorylation activities [4]. Mitophagy can be regulated by different upstream regulators, such as PINK1, TRAP1, and Parkin, to induce cancer cell death [4]. Furthermore, treatment with matrine, a natural alkaloid, modulates the PINK1/Parkin pathways and altered protective mitophagy, thereby provoking mitochondrial apoptosis in HPC HepG2 cells [53]. Our result indicated that PINK1/Parkin was upregulated, whereas TRAP1 was 
downregulated in response to the treatment of E/LPN-KL and miR125/SLN-KL (Figure 4d). This result revealed that the co-treatment of miR-125 and $\mathrm{E}$ in mitochondrion-targeted nanoparticles might regulate mitophagy by modulating the pathways of mitochondrial remodeling (i.e., PINK1/Parkin) and oxidative stress (i.e., TRAP1) in SAS cells. Furthermore, we found OPA1, a mitochondrial fusion marker, was cleaved, but p-Drp1, a mitochondrial fission marker, increased after the treatment of E/LPN-KL and miR125/SLN-KL (Figure 4d). This result suggested that mitochondrial fission was more favorable than fusion to the induction of apoptosis (Figure $6 \mathrm{c}, \mathrm{d}$ ). Moreover, this combination therapy increased the protein levels of LCII, Beclin 1, Atg5, and RIP1/3, indicating that the co-treatment of miR-125 and $\mathrm{E}$ in nanoparticle preparations might regulate mitochondrial autophagy/mitophagy and necropotosis pathways in SAS cells.

Intriguingly, another study has shown that Krüppel-like factor 4, a zinc finger transcription factor, considerably increases ROS levels and triggers mitochondrial fusion, thus inducing the G2/M cell cycle arrest and protecting cells from nutrient deprivation-induced death in human glioblastoma cells [54]. Consistent with our current finding, previous evidence has suggested that excessive mitochondrial fission may happen during cell metabolism, proliferation, apoptosis, and migration through the activation of the intrinsic (mitochondrial) apoptotic pathway [3]. Accordingly, the Drp1 post-transcriptional phosphorylation and alteration of the Parkin transcription activity via p53 may trigger mitochondrial fission and aberrant mitophagy [4]. Such mitophagy defect may provoke cellular oxidative stress, energy metabolism imbalance, and calcium overload; consequently, it induces caspase 9-mediated intrinsic (mitochondrial) apoptosis and abolishes F-actin-dependent cellular migration in cancer cells [6]. Correspondingly, our result implied that the treatment of E/LPN-KL and miR125/SLN-KL considerably suppressed SAS cell migration and MDR by inhibiting $\beta$-catenin, $\mathrm{N}$-cadherin, vimentin, snail, and Slug in the EMT pathway and P-gp and MRP1/2 in the MDR pathway (Figure 5). The mitochondria are responsible for the dynamic equilibrium of various pro-apoptotic and anti-apoptotic proteins, such as Bcl-2 and Bax [43]. Remarkably, the co-treatment of E/LPN-KL and miR125/SLN-KL significantly induced the SAS cell apoptosis by effectively inhibiting Bcl-2 and Mcl-1 and by inducing the cascade cleavage of PARP, caspase-3, caspase-8, and caspase-9 (Figure 6). These results confirmed the potential of E/LPN-KL and miR125/SLN-KL for application in overcoming migration and MDR while enhancing apoptosis and increasing cytotoxicity against SAS cells (Figures 5 and 6). The safety evaluation in NOK cells also suggested the protective effect elicited by LPN-KL nanovehicles to decrease the toxic effects of $\mathrm{E}$ on noncancerous cells (Figure $6 \mathrm{~b}$ ).

Encouraging preclinical findings suggested that the co-treatment of E/LPN-KL and miR125/SLN-KL given to SAS-bearing mice reduced blood glucose and cholesterol levels (Figure 7a,b), indicating that E/LPN-KL and miR125/SLN-KL might modulate the pathways associated with tumor lipid and glucose metabolism and, consequently, disturb tumor progression and growth. Concomitantly, the co-treatment of E/LPN-KL and miR125/SLN-KL reduced the tumor size in SAS-bearing mice compared with that of CTR, E, E/LPN, and E/LPN-KL $(p<0.001-0.01$; Figure $8 \mathrm{a}, \mathrm{b})$. These results validated that targeting EGFR of SAS-tumor cells and effective intracellular and mitochondrial delivery are key prerequisites for lipid nanoparticles, such as LPN-KL and SLN-KL, to display a superior antitumor efficacy and excellent hypolipidemic and hypoglycemic effects in vivo. No obvious changes in the body weight and biochemical markers of the heart, liver, and kidney were observed in the treatment groups (Figures $7 \mathrm{c}-\mathrm{e}$ and $8 \mathrm{~b}$ ). These results suggested the acceptable biosafety of the E- and miR-125-containing formulations. This finding was also consistent with the subchronic toxicity evaluation of $\mathrm{E}$ as a food additive in F344 rats; that is, no death or treatment-associated clinical symptoms were detected during the experimental period [55].

\section{Conclusions}

We developed EGFR-targeting and mitochondrion-directed nanoparticles to incorporate $\mathrm{E}$ and miR-125 for the modulation of lipid and glucose metabolism, mitochondrial oxidative stress, remodeling, bioenergenesis, mitophagy, and fusion/fission dynamics. On the basis of the data about 
mitochondrial respiration and aerobic glycolysis, we demonstrated that the inhibitory effect of miR125/SLN-KL and E/LPN-KL on cellular bioenergetics was highly correlated with their anticancer activity. These formulations also regulated multiple pathways of tumor metabolism, mitochondrial dynamics, apoptosis, resistance, and metastasis to promote the sensitivity of SAS cells to E and miR-125. These dosage forms also elicited low toxic effects on normal cells. To our best knowledge, this prospective study can be used as a basis for combining miR-125 with natural compounds, such as E, in nanoformulations to regulate mitochondrial dynamics and energy metabolism associated with cancer.

Supplementary Materials: The following are available online at http://www.mdpi.com/1999-4923/12/8/756/s1, Table S1: Antibodies used in this study.

Author Contributions: Conceptualization, Y.-L.L., Y.-H.C., and C.-J.C.; Data curation, Y.-L.L., C.-S.W., and Y.-C.C.; Formal analysis, Y.-L.L. and C.-S.W.; Funding acquisition, Y.-L.L., Y.-H.C., and C.-J.C.; Investigation, Y.-L.L., Y.-C.C., T.-Y.W., and C.-P.Y.; Methodology, Y.-L.L., C.-S.W., Y.-C.C., and T.-Y.W.; Project administration, Y.-L.L.; Resources, Y.-L.L.; Supervision, Y.-L.L.; Validation, Y.-L.L. and C.-S.W.; Visualization, Y.-L.L.; Writing-original draft, Y.-L.L.; Writing-review and editing, Y.-L.L. All authors have read and agreed to the published version of the manuscript.

Funding: This research was funded by Veterans General Hospitals and University System of Taiwan Joint Research Program (VGHUST106-G7-7-3; VGHUST108-G2-1-2; VGHUST109-V7-3-2), Ministry of Science and Technology of Taiwan (MOST 106-2320-B-010-005; MOST 107-2320-B-010-015-MY3), National Yang-Ming University and Cheng Hsin General Hospital (CY10839; CY10935), Yin Yen-Liang Foundation Development and Construction Plan of the School of Medicine, Center for Advanced Pharmaceutics and Drug Delivery Research, National Yang-Ming University.

Conflicts of Interest: The authors declare no conflict of interest. The funders had no role in the design of the study; in the collection, analyses, or interpretation of data; in the writing of the manuscript, or in the decision to publish the results.

\section{References}

1. Kao, Y.Y.; Chou, C.H.; Yeh, L.Y.; Chen, Y.F.; Chang, K.W.; Liu, C.J.; Fan Chiang, C.Y.; Lin, S.C. MicroRNA miR-31 targets SIRT3 to disrupt mitochondrial activity and increase oxidative stress in oral carcinoma. Cancer Lett. 2019, 456, 40-48. [CrossRef]

2. Li, S.; Wu, Y.; Ding, Y.; Yu, M.; Ai, Z. CerS6 regulates cisplatin resistance in oral squamous cell carcinoma by altering mitochondrial fission and autophagy. J. Cell Physiol. 2018, 233, 9416-9425. [CrossRef]

3. Wen, R.; Banik, B.; Pathak, R.K.; Kumar, A.; Kolishetti, N.; Dhar, S. Nanotechnology inspired tools for mitochondrial dysfunction related diseases. Adv. Drug Deliv. Rev. 2016, 99, 52-69. [CrossRef]

4. Zhao, Q.; Ye, M.; Yang, W.; Wang, M.; Li, M.; Gu, C.; Zhao, L.; Zhang, Z.; Han, W.; Fan, W.; et al. Effect of Mst1 on Endometriosis Apoptosis and Migration: Role of Drp1-Related Mitochondrial Fission and Parkin-Required Mitophagy. Cell Physiol. Biochem. 2018, 45, 1172-1190. [CrossRef]

5. McWilliams, T.G.; Muqit, M.M. PINK1 and Parkin: emerging themes in mitochondrial homeostasis. Curr. Opin. Cell Biol. 2017, 45, 83-91. [CrossRef]

6. Shefa, U.; Jeong, N.Y.; Song, I.O.; Chung, H.J.; Kim, D.; Jung, J.; Huh, Y. Mitophagy links oxidative stress conditions and neurodegenerative diseases. Neural Regen Res. 2019, 14, 749-756.

7. Zhao, C.; He, R.; Shen, M.; Zhu, F.; Wang, M.; Liu, Y.; Chen, H.; Li, X.; Qin, R. PINK1/Parkin-Mediated Mitophagy Regulation by Reactive Oxygen Species Alleviates Rocaglamide A-Induced Apoptosis in Pancreatic Cancer Cells. Front. Pharm. 2019, 10, 968. [CrossRef]

8. Yao, N.; Wang, C.; Hu, N.; Li, Y.; Liu, M.; Lei, Y.; Chen, M.; Chen, L.; Chen, C.; Lan, P.; et al. Inhibition of PINK1/Parkin-dependent mitophagy sensitizes multidrug-resistant cancer cells to B5G1, a new betulinic acid analog. Cell Death Dis. 2019, 10, 232. [CrossRef]

9. Huang, C.Y.; Kuo, W.W.; Ho, T.J.; Chiang, S.F.; Pai, P.Y.; Lin, J.Y.; Lin, D.Y.; Kuo, C.H.; Huang, C.Y. Rab9-dependent autophagy is required for the IGF-IIR triggering mitophagy to eliminate damaged mitochondria. J. Cell Physiol. 2018, 233, 7080-7091. [CrossRef]

10. He, L.; Gu, K. Tanshinone IIA regulates colorectal cancer apoptosis via attenuation of Parkin-mediated mitophagy by suppressing AMPK/Skp2 pathways. Mol. Med. Rep. 2018, 18, 1692-1703. [CrossRef] 
11. Di Rita, A.; Peschiaroli, A.; Pasquale, D.; Strobbe, D.; Hu, Z.; Gruber, J.; Nygaard, M.; Lambrughi, M.; Melino, G.; Papaleo, E.; et al. HUWE1 E3 ligase promotes PINK1/PARKIN-independent mitophagy by regulating AMBRA1 activation via IKKalpha. Nat. Commun. 2018, 9, 3755. [CrossRef]

12. Yan, C.; Li, T.S. Dual Role of Mitophagy in Cancer Drug Resistance. Anticancer Res. 2018, 38, 617-621.

13. Wang, J.; Gao, S.; Wang, S.; Xu, Z.; Wei, L. Zinc oxide nanoparticles induce toxicity in CAL 27 oral cancer cell lines by activating PINK1/Parkin-mediated mitophagy. Int. J. Nanomed. 2018, 13, 3441-3450. [CrossRef]

14. Yang, J.; Zhang, S.; Henning, S.M.; Lee, R.; Hsu, M.; Grojean, E.; Pisegna, R.; Ly, A.; Heber, D.; Li, Z. Cholesterol-lowering effects of dietary pomegranate extract and inulin in mice fed an obesogenic diet. J. Nutr. Biochem. 2018, 52, 62-69. [CrossRef]

15. Les, F.; Arbones-Mainar, J.M.; Valero, M.S.; Lopez, V. Pomegranate polyphenols and urolithin A inhibit alpha-glucosidase, dipeptidyl peptidase-4, lipase, triglyceride accumulation and adipogenesis related genes in 3T3-L1 adipocyte-like cells. J. Ethnopharmacol. 2018, 220, 67-74. [CrossRef]

16. Panchal, S.K.; Ward, L.; Brown, L. Ellagic acid attenuates high-carbohydrate, high-fat diet-induced metabolic syndrome in rats. Eur. J. Nutr. 2013, 52, 559-568. [CrossRef]

17. Liu, M.P.; Liao, M.; Dai, C.; Chen, J.F.; Yang, C.J.; Liu, M.; Chen, Z.G.; Yao, M.C. Sanguisorba officinalis L synergistically enhanced 5 -fluorouracil cytotoxicity in colorectal cancer cells by promoting a reactive oxygen species-mediated, mitochondria-caspase-dependent apoptotic pathway. Sci. Rep. 2016, 6, 34245. [CrossRef]

18. Shi, C.; Cai, Y.; Li, Y.; Li, Y.; Hu, N.; Ma, S.; Hu, S.; Zhu, P.; Wang, W.; Zhou, H. Yap promotes hepatocellular carcinoma metastasis and mobilization via governing cofilin/F-actin/lamellipodium axis by regulation of JNK/Bnip3/SERCA/CaMKII pathways. Redox Biol. 2018, 14, 59-71. [CrossRef]

19. Wu, Q.B.; Chen, J.; Zhu, J.W.; Yin, X.; You, H.Y.; Lin, Y.R.; Zhu, H.Q. MicroRNA125 inhibits RKO colorectal cancer cell growth by targeting VEGF. Int. J. Mol. Med. 2018, 42, 665-673.

20. Ma, J.; Fan, Y.; Feng, T.; Chen, F.; Xu, Z.; Li, S.; Lin, Q.; He, X.; Shi, W.; Liu, Y.; et al. HOTAIR regulates HK2 expression by binding endogenous miR-125 and miR-143 in oesophageal squamous cell carcinoma progression. Oncotarget 2017, 8, 86410-86422. [CrossRef]

21. Giroud, M.; Pisani, D.F.; Karbiener, M.; Barquissau, V.; Ghandour, R.A.; Tews, D.; Fischer-Posovszky, P.; Chambard, J.C.; Knippschild, U.; Niemi, T.; et al. miR-125b affects mitochondrial biogenesis and impairs brite adipocyte formation and function. Mol. Metab. 2016, 5, 615-625. [CrossRef]

22. Chen, Y.; Gao, D.-Y.; Huang, L. In vivo delivery of miRNAs for cancer therapy: challenges and strategies. Adv. Drug Deliv. Rev. 2015, 81, 128-141. [CrossRef]

23. Dhingra, A.; Jayas, R.; Afshar, P.; Guberman, M.; Maddaford, G.; Gerstein, J.; Lieberman, B.; Nepon, H.; Margulets, V.; Dhingra, R.; et al. Ellagic acid antagonizes Bnip3-mediated mitochondrial injury and necrotic cell death of cardiac myocytes. Free Radic. Biol. Med. 2017, 112, 411-422. [CrossRef]

24. Hong, S.T.; Lin, H.; Wang, C.S.; Chang, C.H.; Lin, A.M.; Yang, J.C.; Lo, Y.L. Improving the anticancer effect of afatinib and microRNA by using lipid polymeric nanoparticles conjugated with dual $\mathrm{pH}$-responsive and targeting peptides. J. Nanobiotechnol. 2019, 17, 89. [CrossRef]

25. Du, J.B.; Song, Y.F.; Ye, W.L.; Cheng, Y.; Cui, H.; Liu, D.Z.; Liu, M.; Zhang, B.L.; Zhou, S.Y. PEG-detachable lipid-polymer hybrid nanoparticle for delivery of chemotherapy drugs to cancer cells. Anticancer Drugs 2014, 25, 751-766. [CrossRef]

26. Ashizawa, A.T.; Cortes, J. Liposomal delivery of nucleic acid-based anticancer therapeutics: BP-100-1.01. Expert Opin. Drug Deliv. 2015, 12, 1107-1120. [CrossRef]

27. Juang, V.; Chang, C.H.; Wang, C.S.; Wang, H.E.; Lo, Y.L. pH-Responsive PEG-Shedding and Targeting Peptide-Modified Nanoparticles for Dual-Delivery of Irinotecan and microRNA to Enhance Tumor-Specific Therapy. Small 2019, 15, e1903296. [CrossRef]

28. Han, C.Y.; Yue, L.L.; Tai, L.Y.; Zhou, L.; Li, X.Y.; Xing, G.H.; Yang, X.G.; Sun, M.S.; Pan, W.S. A novel small peptide as an epidermal growth factor receptor targeting ligand for nanodelivery in vitro. Int. J. Nanomed. 2013, 8, 1541-1549.

29. Jiang, L.; Li, L.; He, X.; Yi, Q.; He, B.; Cao, J.; Pan, W.; Gu, Z. Overcoming drug-resistant lung cancer by paclitaxel loaded dual-functional liposomes with mitochondria targeting and $\mathrm{pH}$-response. Biomaterials 2015, 52, 126-139. [CrossRef]

30. Kim, J.Y.; Han, J.H.; Park, G.; Seo, Y.W.; Yun, C.W.; Lee, B.C.; Bae, J.; Moon, A.R.; Kim, T.H. Necrosis-inducing peptide has the beneficial effect on killing tumor cells through neuropilin (NRP-1) targeting. Oncotarget 2016, 7, 32449-32461. [CrossRef] 
31. Meschenmoser, K.; Kim, Y.; Franken, S.; Nowak, M.; Feldmann, G.; Bendas, G.; Wolfgarten, M.; Messmer, D.; Schmidt-Wolf, I.G. Targeting cancer with a bi-functional peptide: in vitro and in vivo results. In Vivo 2013, 27, 431-442.

32. He, C.; Jiang, S.; Jin, H.; Chen, S.; Lin, G.; Yao, H.; Wang, X.; Mi, P.; Ji, Z.; Lin, Y.; et al. Mitochondrial electron transport chain identified as a novel molecular target of SPIO nanoparticles mediated cancer-specific cytotoxicity. Biomaterials 2016, 83, 102-114. [CrossRef]

33. Zhang, D.M.; Shu, C.; Chen, J.J.; Sodani, K.; Wang, J.; Bhatnagar, J.; Lan, P.; Ruan, Z.X.; Xiao, Z.J.; Ambudkar, S.V.; et al. BBA, a Derivative of 23-Hydroxybetulinic Acid, Potently Reverses ABCB1-Mediated Drug Resistance in Vitro and in Vivo. Mol. Pharm. 2012, 9, 3147-3159. [CrossRef]

34. Tang, Q.Q.; Otto, T.C.; Lane, M.D. CCAAT/enhancer-binding protein beta is required for mitotic clonal expansion during adipogenesis. Proc. Natl. Acad. Sci. USA 2003, 100, 850-855. [CrossRef]

35. Domori, A.; Sunahara, A.; Tateno, M.; Miyama, T.S.; Setoguchi, A.; Endo, Y. The clinical utility of two human portable blood glucose meters in canine and feline practice. Vet. Clin. Pathol. 2014, 43, 55-62. [CrossRef]

36. Ma, D. Enhancing endosomal escape for nanoparticle mediated siRNA delivery. Nanoscale 2014, 6, 6415-6425. [CrossRef]

37. Wood Dos Santos, T.; Cristina Pereira, Q.; Teixeira, L.; Gambero, A.; A Villena, J.; Lima Ribeiro, M. Effects of Polyphenols on Thermogenesis and Mitochondrial Biogenesis. Int. J. Mol. Sci 2018, 19, 2757. [CrossRef]

38. He, W.; Zhang, A.; Qi, L.; Na, C.; Jiang, R.; Fan, Z.; Chen, J. FOXO1, a Potential Therapeutic Target, Regulates Autophagic Flux, Oxidative Stress, Mitochondrial Dysfunction, and Apoptosis in Human Cholangiocarcinoma QBC939 Cells. Cell. Physiol. Biochem. Int. J. Exp. Cell. Physiol. Biochem. Pharmacol. 2018, 45, 1506-1514. [CrossRef]

39. Hohnholt, M.C.; Andersen, V.H.; Bak, L.K.; Waagepetersen, H.S. Glucose, Lactate and Glutamine but not Glutamate Support Depolarization-Induced Increased Respiration in Isolated Nerve Terminals. Neurochem. Res. 2017, 42, 191-201. [CrossRef]

40. Li, L.; Chen, Q.; Yu, Y.; Chen, H.; Lu, M.; Huang, Y.; Li, P.; Chang, H. RKI-1447 suppresses colorectal carcinoma cell growth via disrupting cellular bioenergetics and mitochondrial dynamics. J. Cell Physiol. 2020, 235, 254-266. [CrossRef]

41. Abdelkader, N.F.; Elyamany, M.; Gad, A.M.; Assaf, N.; Fawzy, H.M.; Elesawy, W.H. Ellagic acid attenuates liver toxicity induced by valproic acid in rats. J. Pharm. Sci. 2020, 143, 23-29. [CrossRef] [PubMed]

42. Tran, Q.; Lee, H.; Park, J.; Kim, S.H.; Park, J. Targeting Cancer Metabolism - Revisiting the Warburg Effects. Toxicol. Res. 2016, 32, 177-193. [CrossRef] [PubMed]

43. Lu, P.; Bruno, B.J.; Rabenau, M.; Lim, C.S. Delivery of drugs and macromolecules to the mitochondria for cancer therapy. J. Control. Release 2016, 240, 38-51. [CrossRef] [PubMed]

44. Sorice, A.; Siano, F.; Capone, F.; Guerriero, E.; Picariello, G.; Budillon, A.; Ciliberto, G.; Paolucci, M.; Costantini, S.; Volpe, M.G. Potential Anticancer Effects of Polyphenols from Chestnut Shell Extracts: Modulation of Cell Growth, and Cytokinomic and Metabolomic Profiles. Molecules 2016, 21, 1411. [CrossRef] [PubMed]

45. Chen, Y.F.; Wei, Y.Y.; Yang, C.C.; Liu, C.J.; Yeh, L.Y.; Chou, C.H.; Chang, K.W.; Lin, S.C. miR-125b suppresses oral oncogenicity by targeting the anti-oxidative gene PRXL2A. Redox Biol. 2019, 22, 101140. [CrossRef]

46. Jiang, J.X.; Gao, S.; Pan, Y.Z.; Yu, C.; Sun, C.Y. Overexpression of microRNA-125b sensitizes human hepatocellular carcinoma cells to 5-fluorouracil through inhibition of glycolysis by targeting hexokinase II. Mol. Med. Rep. 2014, 10, 995-1002. [CrossRef]

47. Li, Y.; Kasim, V.; Yan, X.; Li, L.; Meliala, I.T.S.; Huang, C.; Li, Z.; Lei, K.; Song, G.; Zheng, X.; et al. Yin Yang 1 facilitates hepatocellular carcinoma cell lipid metabolism and tumor progression by inhibiting PGC-1 $\beta$-induced fatty acid oxidation. Theranostics 2019, 9, 7599-7615. [CrossRef]

48. Cheng, K.T.; Wang, Y.S.; Chou, H.C.; Chang, C.C.; Lee, C.K.; Juan, S.H. Kinsenoside-mediated lipolysis through an AMPK-dependent pathway in C3H10T1/2 adipocytes: Roles of AMPK and PPARalpha in the lipolytic effect of kinsenoside. Phytomedicine 2015, 22, 641-647. [CrossRef]

49. Ruan, W.; Li, J.; Xu, Y.; Wang, Y.; Zhao, F.; Yang, X.; Jiang, H.; Zhang, L.; Saavedra, J.M.; Shi, L.; et al. MALAT1 Up-Regulator Polydatin Protects Brain Microvascular Integrity and Ameliorates Stroke through C/EBPbeta/MALAT1/CREB/PGC-1alpha/PPARgamma Pathway. Cell Mol. Neurobiol. 2019, 39, 265-286. [CrossRef] 
50. Woo, M.S.; Choi, H.S.; Seo, M.J.; Jeon, H.J.; Lee, B.Y. Ellagic acid suppresses lipid accumulation by suppressing early adipogenic events and cell cycle arrest. Phytother. Res. 2015, 29, 398-406. [CrossRef]

51. Lv, O.; Wang, L.; Li, J.; Ma, Q.; Zhao, W. Effects of pomegranate peel polyphenols on lipid accumulation and cholesterol metabolic transformation in L-02 human hepatic cells via the PPARgamma-ABCA1/CYP7A1 pathway. Food Funct. 2016, 7, 4976-4983. [CrossRef] [PubMed]

52. Piccinin, E.; Peres, C.; Bellafante, E.; Ducheix, S.; Pinto, C.; Villani, G.; Moschetta, A. Hepatic peroxisome proliferator-activated receptor $\gamma$ coactivator $1 \beta$ drives mitochondrial and anabolic signatures that contribute to hepatocellular carcinoma progression in mice. Hepatology 2018, 67, 884-898. [CrossRef] [PubMed]

53. Wei, R.; Cao, J.; Yao, S. Matrine promotes liver cancer cell apoptosis by inhibiting mitophagy and PINK1/Parkin pathways. Cell Stress Chaperones 2018, 23, 1295-1309. [CrossRef] [PubMed]

54. Wang, S.; Shi, X.; Wei, S.; Ma, D.; Oyinlade, O.; Lv, S.Q.; Ying, M.; Zhang, Y.A.; Claypool, S.M.; Watkins, P.; et al. Kruppel-like factor 4 (KLF4) induces mitochondrial fusion and increases spare respiratory capacity of human glioblastoma cells. J. Biol. Chem. 2018, 293, 6544-6555. [CrossRef] [PubMed]

55. Tasaki, M.; Umemura, T.; Maeda, M.; Ishii, Y.; Okamura, T.; Inoue, T.; Kuroiwa, Y.; Hirose, M.; Nishikawa, A. Safety assessment of ellagic acid, a food additive, in a subchronic toxicity study using F344 rats. Food Chem. Toxicol. 2008, 46, 1119-1124. [CrossRef]

(C) 2020 by the authors. Licensee MDPI, Basel, Switzerland. This article is an open access article distributed under the terms and conditions of the Creative Commons Attribution (CC BY) license (http://creativecommons.org/licenses/by/4.0/). 